\section{A) Check for updates}

Cite this: Dalton Trans., 2021, 50 , 13786

Received 26th July 2021 Accepted 6th September 2021

DOI: $10.1039 / d 1 d t 02474 d$

rsc.li/dalton

\title{
Evaluation of $\mathrm{Ga}_{0.2} \mathrm{Li}_{6.4} \mathrm{Nd}_{3} \mathrm{Zr}_{2} \mathrm{O}_{12}$ garnets: exploit- ing dopant instability to create a mixed conductive interface to reduce interfacial resistance for all solid state batteries $\uparrow$
}

\author{
M. P. Stockham, (D) *a B. Dong, (D) a M. S. James, ${ }^{a}$ Y. Li, ${ }^{\text {b }}$ Y. Ding, ${ }^{b}$ E. Kendrick (D) ${ }^{c}$ and \\ P. R. Slater (iD *a
}

\begin{abstract}
The next major leap in energy storage is thought to arise from a practical implementation of all solid-state batteries, which remain largely confined to the small scale due to issues in manufacturing and mechanical stability. Lithium batteries are amongst the most sought after, for the high expected energy density and improved safety characteristics, however the challenge of finding a suitable solid-state electrolyte remains. Lithium rich garnets are prime contenders as electrolytes, owing to their high ionic conductivity $\left(>0.1 \mathrm{mS} \mathrm{cm}^{-1}\right)$, wide electrochemical window $(0-6 \mathrm{~V})$ and stability with Li metal. However, the high Young's modulus of these materials, poor wetting of $\mathrm{Li}$ metal and rapid formation of $\mathrm{Li}_{2} \mathrm{CO}_{3}$ passivating layers tends to give a detrimentally large resistance at the solid-solid interface, limiting their application in solid state batteries. Most studies have focused on La based systems, with very little work on other lanthanides. Here we report a study of the $\mathrm{Nd}$ based garnet $\mathrm{Ga}_{0.2} \mathrm{Li}_{6.4} \mathrm{Nd}_{3} \mathrm{Zr}_{2} \mathrm{O}_{12}$, illustrating substantial differences in the interfacial behaviour. This garnet shows very low interfacial resistance attributed to dopant exsolution which, when combined with moderate heating $\left(175^{\circ} \mathrm{C}, 1 \mathrm{~h}\right)$ with Li metal, we suggest forms $\mathrm{Ga}$-Li eutectics, which significantly reduces the resistance at the Li/garnet interface to as low as $67 \Omega \mathrm{cm}^{2}$ (much lower than equivalent La based systems). The material also shows intrinsically high density (93\%) and good conductivity $\left(\geq 0.2 \mathrm{mS} \mathrm{cm}{ }^{-1}\right)$ via conventional furnaces in air. It is suggested these garnets are particularly well suited to provide a mixed conductive interface (in combination with other garnets) which could enable future solid-state batteries.
\end{abstract}

\section{Introduction}

Lithium ion batteries (LIBs) are the electrochemical system of choice for many energy storage applications. To further increase energy density the move to lithium metal negative electrodes, or anodes, is required. This is a particular challenge for cells containing liquid based electrolytes due to hazardous dendritic lithium growth and numerous safety implications. ${ }^{1-3}$ Liquid electrolytes are also toxic, flammable, and unable to be employed with high $\mathrm{V}$ cathode materials due to their relatively narrow electrochemical window. ${ }^{4-9}$

\footnotetext{
${ }^{a}$ School of Chemistry, University of Birmingham, Birmingham B15 2TT, UK. E-mail: mps846@bham.ac.uk

${ }^{b}$ School of Chemical Engineering, University of Birmingham, Birmingham B15 2TT, UK

${ }^{c}$ School of Metallurgy and Materials, University of Birmingham, Birmingham B15 2TT, UK

$\dagger$ Electronic supplementary information (ESI) available. See DOI: 10.1039/ d1dt02474d
}

Therefore, the replacement of these electrolytes is a pre-requisite for the next major leap in energy storage. ${ }^{4,10-13}$

Solid state batteries (SSB) enable safer, higher energy density and (theoretically, assuming a perfectly dense structure) are able to block Li dendrite growth, however a practical solid state electrolyte (SSE) remains an intense research challenge. ${ }^{14-16}$ Most SSEs, based on oxide and sulphide chemistries, often have either a poor electrochemical window or low ionic conductivity, while also requiring time consuming, energy demanding and/or complex synthesis routes. ${ }^{17-25}$ Furthermore, many SSEs also suffer from large interfacial resistance due to poor wetting at the solid-solid interface, thus limiting the practical use of many SSBs. ${ }^{25-28}$

Amongst the oxide SSEs, lithium rich garnet materials have seen enormous interest due to their high room temperature conductivity (rivalling liquid electrolytes at $>0.1 \mathrm{mS} \mathrm{cm}^{-1}$ ), a wide electrochemical window $(0-6 \quad \mathrm{~V})$ and stability with lithium metal. ${ }^{18,29-35}$ This, therefore, positions these garnet materials relatively uniquely amongst other SSEs, making them promising SSB candidates. 
Typical garnets have the general formula $\mathrm{A}_{3} \mathrm{~B}_{2} \mathrm{X}_{3} \mathrm{O}_{12}$ (e.g. $\mathrm{A}=$ $\mathrm{Fe}, \mathrm{Mg}, \mathrm{B}=\mathrm{Al}, \mathrm{Cr}, \mathrm{Fe}$, and $\mathrm{X}=\mathrm{Si}, \mathrm{Fe}, \mathrm{Al}, \mathrm{Ga}) \cdot{ }^{36-39}$ An example of such a $\mathrm{Li}_{3}$ based garnet system is $\mathrm{Li}_{3} \mathrm{Nd}_{3} \mathrm{~W}_{2} \mathrm{O}_{12}$, with lithium fully occupying the $24 d$ tetrahedral site, although this garnet shows minimal Li ion mobility. ${ }^{38,40}$ Through modifying this system with lower valent cations, increased Li content results in order to maintain charge neutrality. This gives increased conductivity, with lithium occupying additional interstitial octahedral sites. ${ }^{37,39,41,42}$ At the upper maximum of $7 \mathrm{Li}$ per formula unit (pfu), Li ordering occurs to reduce short Li-Li distances as all Li sites are fully occupied, which gives an elongation of an axis in the cubic cell. Therefore, Li garnets present with two distinct crystal systems: cubic and tetragonal. ${ }^{43-47}$ Tetragonal garnets have $7 \mathrm{Li} \mathrm{pfu}$ and poor conductivity with the $I 4_{1} / A C D$ space group (no. 142). ${ }^{43-46}$ Conversely cubic garnets have $<7 \mathrm{Li}$ pfu and show high conductivity and either $I a \overline{3} d$ (no. 230) or $I \overline{4} 3 d$ (no. 220) symmetry. ${ }^{39,48,49}$ Lithium garnets are usually most conductive when the Li content is between $\sim 6.2-6.6$, as some vacant interstitial sites are required to provide an effective migration pathway. ${ }^{24,29,32,34,37,41-43,50-63}$

One of the garnet systems that has attracted considerable attention is $\mathrm{Li}_{7-3 x} \mathrm{Ga}_{x} \mathrm{La}_{3} \mathrm{Zr}_{2} \mathrm{O}_{12}$ (Ga-LLZO), ${ }^{29,55,59,64}$ however, there are still challenges with this system to achieve the highest conductivity, which typically requires the powder to be fully synthesised/processed under argon or $\mathrm{O}_{2}$ to enable high densities and minimize atmospheric proton exchange. ${ }^{29}$ Similarly complex procedures are often required to reduce the high interfacial resistance encountered at the interface with the Li metal anode, which is currently one of the key limiting factors toward practical garnet battery applications. This issue is compounded by the rapid formation of a $\mathrm{Li}_{2} \mathrm{CO}_{3}$ passivating layer, with reports suggesting it forms within minutes of air exposure. ${ }^{65-67}$ Therefore, a need arises to investigate other potential garnet systems which can offer improved conductivity, densification and, crucially, intrinsically low interfacial resistance.

Several reports have previously discussed the potential instability of $\mathrm{Al}$ and $\mathrm{Ga}$ doped LLZO, with dopant exsolution shown to plate the grain boundaries in oxide form after densification. ${ }^{53,68}$ Although this has been shown to be advantageous for pellet densification, it also creates Li deficient grain boundaries which can impede overall ionic conductivity. Dopant exsolution, however, has yet to be explored as a potential way to decrease interfacial resistance at the Li-garnet interface. This could be accomplished by enhancing dopant exsolution to enable alloying with $\mathrm{Li}$ metal, particularly with $\mathrm{Ga}$ doped garnets in the form of Ga-Li eutectics. In doing so, this could create a low impedance interface and remove the need for additional coatings/interlayers/complex techniques post SSE membrane formation. Most reports of dopant exsolution relate more to the $\mathrm{Al}$ substituted garnets rather than $\mathrm{Ga}$, however the latter is far more favourable to form $\mathrm{Li}$ based eutectics at temperatures $>28{ }^{\circ} \mathrm{C} .{ }^{69-71}$

Therefore, to examine this possibility a Ga doped garnet must be designed which has enhanced dopant site instability (to enable increased exsolution). In this work, we examine whether replacing La by Nd can generate such an effect, as a result of strain created by the reduced lattice parameters associated with the smaller $\mathrm{Nd}^{3+}$ ion. As Ga-Li eutectics form above $28^{\circ} \mathrm{C}$, subsequent heating of a $\mathrm{Ga}$ doped $\mathrm{Nd}$ garnet in the presence of Li metal may enable enhanced site instability (due to reactivity of Li) and favour formation of the eutectic alloy. ${ }^{69,71}$

Herein, we report the synthesis of $\mathrm{Li}_{6.4} \mathrm{Ga}_{0.2} \mathrm{Nd}_{3} \mathrm{Zr}_{2} \mathrm{O}_{12}$ (GaNLZO) for the first time, which demonstrates Ga-exsolution which has been considerably enhanced post Li symmetry cell formation, when compared to reports of Ga-LLZO/GaLLHO. ${ }^{32,55}$ Significantly Ga-NLZO demonstrates exceptionally low interfacial resistance of $67 \Omega \mathrm{cm}^{2}$ intrinsic to the garnet material and pellets/powder can be prepared in air. Furthermore, sintered Ga-NLZO has high density (93\%), good room temperature conductivity $\left(\sim 0.3 \mathrm{mS} \mathrm{cm}^{-1}\right)$, a wide electrochemical window and cycling stability.

To ascertain if Ga-Li alloying occurs we have performed post cycling analysis and investigate the reaction between purchased $\mathrm{Ga}_{2} \mathrm{O}_{3}$ and Li metal under similar circumstances. GaNLZO is shown to have promising applications as a conductive interface (CI) with proof-of-concept lithium garnet "sandwich" type cells constructed in the form of Ga-NLZO/ $\mathrm{Li}_{6.5} \mathrm{La}_{3} \mathrm{Zr}_{1.25} \mathrm{Ce}_{0.25} \mathrm{Ta}_{0.5} \mathrm{O}_{12}$ (Ta-LLZCO)/Ga-NLZO. As there have been few reports of $\mathrm{Nd}$ based garnet systems $\mathrm{s}^{3,38,72}$ we also extended this study to structural and impedance evaluation of $\mathrm{Li}_{5+x} \mathrm{Nd}_{3} \mathrm{Nb}_{2-x} \mathrm{Zr}_{x} \mathrm{O}_{12}(0 \leq x \geq 2)$ and $\mathrm{Li}_{6.4} \mathrm{Al}_{0.2} \mathrm{Nd}_{3} \mathrm{Zr}_{2} \mathrm{O}_{12}$ (AlNLZO).

\section{Methods}

\section{Synthesis}

$\mathrm{Li}_{5+x} \mathrm{La}_{3} \mathrm{Nb}_{2-x} \mathrm{Zr}_{x} \mathrm{O}_{12}(0 \leq x \geq 2)$ and $\mathrm{Li}_{6.4} \mathrm{Ga}_{0.2} / \mathrm{Al}_{0.2} \mathrm{Nd}_{3} \mathrm{Zr}_{2} \mathrm{O}_{12}$ were prepared via the solid-state route from stochiometric quantities of $\mathrm{Li}_{2} \mathrm{CO}_{3}, \mathrm{Nd}_{2} \mathrm{O}_{3}, \mathrm{Nb}_{2} \mathrm{O}_{5}, \mathrm{ZrO}_{2}, \mathrm{Al}_{2} \mathrm{O}_{3}$ and $\mathrm{Ga}_{2} \mathrm{O}_{3}$ in air. A $20-40 \%$ mol excess of lithium was added to compensate for lithium loss during high temperature sintering. All powders were ball milled for $1 \mathrm{~h}$ with $\mathrm{ZrO}_{2}$ balls $(500 \mathrm{rpm})$ with hexane, pressed into pellets and heated to $1050{ }^{\circ} \mathrm{C}$ (6 hours) at $5{ }^{\circ} \mathrm{C} \mathrm{min}^{-1}$. Post sintering, pellets were sanded to remove any $\mathrm{Al}$ contamination from the $\mathrm{Al}$ crucible. Al-NLZO and $\mathrm{Li}_{6.4} \mathrm{Nd}_{3} \mathrm{Nb}_{0.6} \mathrm{Zr}_{1.4} \mathrm{O}_{12}$ required a second heat treatment with $20 \%$ wt. Li excess to achieve single phase samples. $\mathrm{Li}_{6.5} \mathrm{La}_{3} \mathrm{Zr}_{1.25} \mathrm{Ce}_{0.25} \mathrm{Ta}_{0.5} \mathrm{O}_{12}$ (Ta-LLZCO) was synthesised similarly (using $\left.\mathrm{CeO}_{2}\right)$, but at $950{ }^{\circ} \mathrm{C}(12 \mathrm{~h})$, for use in the layered cells as previous reports suggest Ce assists with low interfacial resistance, hence may assist in the layered structure. ${ }^{47}$

\section{Characterisation}

All samples were stored in an argon glove box to prevent proton-Li exchange in the garnet, which is also thought to occur in Nd based garnets. ${ }^{3,73}$ Scanning electron microscopy (SEM) was performed on a Hitachi TM4000plus instrument, with the elemental distribution confirmed by the corres- 
ponding AZtecOne energy dispersive X-ray (EDX) attachment. Samples were prepared by applying the powders to a carbon tape and analysed at $15 \mathrm{kV}$ in backscattered electron mode. Pellets were also examined in this manner, which were polished with silicon carbide sandpaper from 240 to 4000 grit to form a flat surface. Phase analysis was performed by X-ray diffraction (XRD) using a Bruker D8 diffractometer with a $\mathrm{Cu}$ $\mathrm{X}$-ray source. Experimental pellet densities were determined and compared to theoretical values from Rietveld refinement results (performed using GSAS II software). ${ }^{74}$

\section{Impedance spectroscopy}

All Nd garnets (for impedance spectroscopy and cell testing) were pelletised and densified in a dry room with a dewpoint between $-45{ }^{\circ} \mathrm{C}$ to $-64{ }^{\circ} \mathrm{C}$ (the elimination of humidity was found to be necessary to prepare good quality samples: it is known that moisture can be an issue in the synthesis of $\mathrm{Li}$ garnet systems). ${ }^{75-77}$ Pellets were prepared as follows: approx. $10 \mathrm{~mm}$ diameter pellets were pressed to $c a$. 1.5 tonnes and heated to $1150{ }^{\circ} \mathrm{C}$ for 13 hours $\left(1.9{ }^{\circ} \mathrm{C} \mathrm{min}^{-1}\right)$. In all cases sacrificial powders were used to protect pellets from $\mathrm{Al}$ contamination via the $\mathrm{Al}_{2} \mathrm{O}_{3}$ crucible and to minimize Li loss. In order to examine the potential of Ga-NLZO as a $\mathrm{Li}$ metal contact layer, layered Ga-NLZO pellets, in the form of GaNLZO/Ta-LLZCO/Ga-NLZO, were prepared in a similar manner, but at $0.4{ }^{\circ} \mathrm{C} \mathrm{min}^{-1}$. Post-sintering, the pellets were polished and were sputtered with $\mathrm{Au}$ for room temperature impedance spectroscopy measurements, which were performed with a Solartron 1260 impedance analyser from $1 \mathrm{~Hz}$ to $10 \mathrm{MHz}$ with a $20 \mathrm{mV}$ potential. Variable temperature measurements were undertaken by painting the polished pellet with $\mathrm{Au}$ electrodes, which required heating to $800{ }^{\circ} \mathrm{C}$ for 1 hour in air to cure the $\mathrm{Au}$ paste. The pellets were then air quenched to room temperature from $>700{ }^{\circ} \mathrm{C}$ (to limit $\mathrm{H} / \mathrm{Li}$ exchange ${ }^{56,78,79}$ ). The variable temperature impedance measurements were measured from 5 $\mathrm{Hz}$ to $13 \mathrm{MHz}$ using a Hewlett Packard 4192A LF instrument with a $100 \mathrm{mV}$ applied potential from $50-143{ }^{\circ} \mathrm{C}$.

\section{Cell assembly}

All cell tests were performed on a biologic VMP3 or SP50 instrument. Li|Ga-NLZO|Li symmetric cells were assembled in an Ar glove box. Firstly, the pellets ( $\sim 1.7 \mathrm{~mm}$ thickness) were polished using silicon carbide sandpaper from 240 to 4000 grit, then lithium metal foil was applied to each side of the pellet. The cell was then heated to $175^{\circ} \mathrm{C}$ under a constant pressure for $1 \mathrm{~h}$ using a commercially and readily available steel 3-way G clamp (see ESI $\dagger$ ) and were secured under light pressure via hand tightening the clamp. The cell was subsequently secured within a Swagelok type cell. Cells were examined via impedance spectroscopy before (and after) cell testing from $10 \mathrm{MHz}$ to $0.1 \mathrm{~Hz}$ with a $20 \mathrm{mV}$ potential on a Solartron 1260 impedance analyser. These symmetric cells were then cycled under constant current conditions at various current densities to assess cycling stability at room temperature on an open lab bench (between $15-21^{\circ} \mathrm{C}$ throughout the day) as well as at a constant temperature $\left(50^{\circ} \mathrm{C}\right)$ in a fire-proof thermal abuse test chamber (MTI). Cells were also cycled with increasing current density, in increments of $10 \mu \mathrm{A} \mathrm{cm} \mathrm{cm}^{-2}$, until a short circuit formed to assess CCD at room temperature on an open lab bench. The ASR was analysed as a function of temperature via impedance measurements for Ga-NLZO. Li/Ga-NLZO/TaLLZCO/Ga-NLZO/Li sandwich type cells were assembled similarly, with $\mathrm{Li}$ stripping and plating assessed on an open lab bench $\left(15-27^{\circ} \mathrm{C}\right)$ and at $43^{\circ} \mathrm{C}$ (to confirm voltage stability).

$\mathrm{Au}|\mathrm{Ga}-\mathrm{NLZO}| \mathrm{Li}$ cells, for cyclic voltammetry (C.V.), were formed by polishing a NLZO pellet as above and hand pressing the pellet into Li foil in an Ar glovebox. The cell was placed in a Swagelok cell with Au foil as the working electrode. Cyclic voltammetry was subsequently run from -0.4 to $5 \mathrm{~V}$ at a scan rate of $1 \mathrm{mV} \mathrm{s}^{-1}$.

\section{Post cycling analysis}

Post cycled Ga-NLZO pellets, which had short circuited, were analysed by SEM and EDX and compared to freshly prepared pellets. For SEM/EDX, pellets were thermally etched in a manner similar to the densification steps above and analysed on the same SEM/EDX instrumentation as above (see ESI $\dagger$ for pellet analysis prior to thermal etching). Cross sectional areas were also analysed via SEM/EDX after the pellet was snapped into smaller segments. The pellet surface and re-ground powder were also analysed via XRD.

\section{Results and discussion}

\section{$\mathrm{X}$-Ray diffraction results}

The powder XRD patterns of $\mathrm{Li}_{5+x} \mathrm{Nd}_{3} \mathrm{Nb}_{2-x} \mathrm{Zr}_{x} \mathrm{O}_{12}(0 \leq x \geq 2)$, $\mathrm{Ga} / \mathrm{Al}-\mathrm{NLZO}$ and $\mathrm{Li}_{7} \mathrm{Nd}_{3} \mathrm{Zr}_{2} \mathrm{O}_{12}$ (NLZO) are shown in Fig. 1. All patterns correspond to the expected garnet symmetry and could be indexed on a cubic Ia $\overline{3} d\left(\mathrm{Li}_{5+x} \mathrm{Nd}_{3} \mathrm{Nb}_{2-x} \mathrm{Zr}_{x} \mathrm{O}_{12} / \mathrm{Al}-\right.$

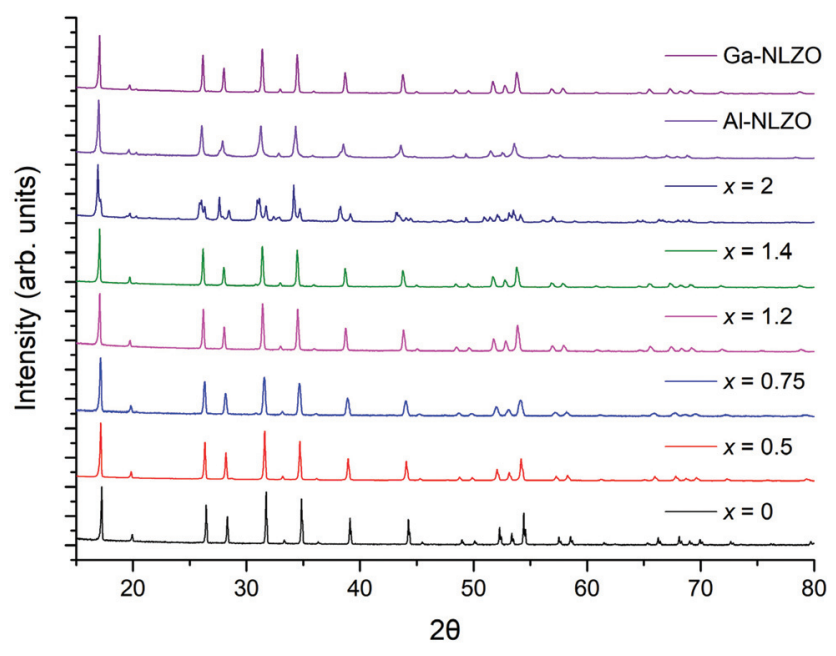

Fig. 1 Powder XRD patterns of $\mathrm{Li}_{5+x} \mathrm{Nd}_{3} \mathrm{Nb}_{2-x} \mathrm{Zr}_{x} \mathrm{O}_{12}(0 \leq x \geq 2)$ and $\mathrm{Li}_{6.4} \mathrm{Al}_{0.2} \mathrm{Nd}_{3} \mathrm{Zr}_{2} \mathrm{O}_{12}$ indexed on la $\overline{3} d$ type symmetry and $\mathrm{Li}_{6.4} \mathrm{Ga}_{0.2} \mathrm{Nd}_{3} \mathrm{Zr}_{2} \mathrm{O}_{12}$ on $1 \overline{4} 3 \mathrm{~d}$ symmetry (see ESI $\uparrow$ for magnified pattern at $\sim 222 \theta$ ). The small peak $\sim 202 \theta$ for Al-NLZO and $x=2$ is attributed to a small $\mathrm{Li}_{2} \mathrm{ZrO}_{3}$ impurity. 
NLZO) or $I \overline{4} 3 d$ (Ga-NLZO) cell. ${ }^{39,44,48}$ All Nd garnets were blue under artificial lighting but instantly turned neodymium glass purple when placed in sunlight, corresponding to the Alexandrite effect as noted previously. ${ }^{80}$

Rietveld refinements on the $\mathrm{Li}_{5+x} \mathrm{Nd}_{3} \mathrm{Nb}_{2-x} \mathrm{Zr}_{x} \mathrm{O}_{12}(0 \leq x \geq$ 1.4) systems were based on the structural model by Cussen ${ }^{39}$ and showed a linear increase in lattice parameters (see Fig. 2), in agreement with Vegard's law, thus further confirming the solid solution range. This increase is attributed to the larger ionic radius of $\mathrm{Zr}^{4+}$ over $\mathrm{Nb}^{5+}$. Lattice parameters were smaller than the La counterparts reported elsewhere, consistent with the smaller radius of $\mathrm{Nd}^{3+} v s$. $\mathrm{La}^{3+}$. Fractional occupancies of $\mathrm{Zr}$ and $\mathrm{Nb}$ were set to the intended ratio, as the similarities in scattering factors preclude accurate refinement using XRD alone (see ESI $\uparrow$ for example refinement). For $x=1.4 \mathrm{small} \mathrm{Nd} /$ $\mathrm{Zr}$ based impurities were present which were unable to be removed. These worsened significantly for samples with $x=$ 1.6 and 1.8 (see ESI $\dagger$ ).

The Ga-NLZO and Al-NLZO samples had larger lattice parameters compared to $\mathrm{Li}_{6.4} \mathrm{Nd}_{3} \mathrm{Zr}_{1.4} \mathrm{Nb}_{0.6} \mathrm{O}_{12}$, see Fig. 1 and 2, with much higher conductivity and improved density for Ga-

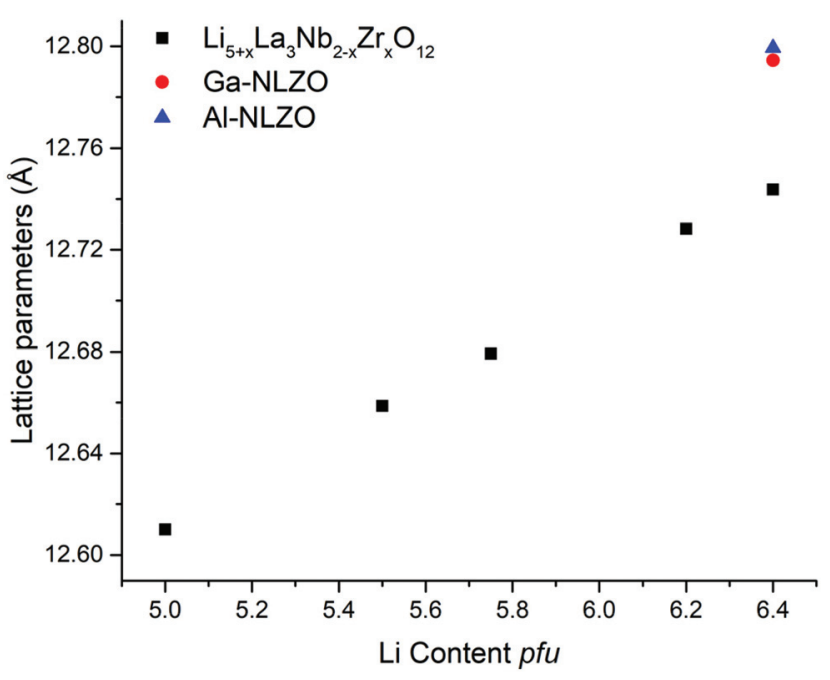

Fig. 2 Lattice parameters vs. Li content of $\mathrm{Li}_{5+x} \mathrm{La}_{3} \mathrm{Nb}_{2-x} \mathrm{Zr}_{x} \mathrm{O}_{12}(0 \leq x \geq$ 1.4), $\mathrm{Li}_{6.4} \mathrm{Al}_{0.2} \mathrm{Nd}_{3} \mathrm{Zr}_{2} \mathrm{O}_{12}$ and $\mathrm{Li}_{6.4} \mathrm{Ga}_{0.2} \mathrm{Nd}_{3} \mathrm{Zr}_{2} \mathrm{O}_{12}$, as determined from Rietveld refinements.
NLZO (see later). These lattice parameters are still far smaller, however, than Ga-LLZO and Ga-LLHO due to the smaller size of $\mathrm{Nd}^{3+} \cdot{ }^{29,32,55}$ For Ga-NLZO both the $I a \overline{3} d$ and $I \overline{4} 3 d$ space groups were considered. The sample has an additional peak $\sim 22^{\circ} 2 \theta$ which is attributed to the acentric cubic space group $I \overline{4} 3 d$, therefore it was assumed to adopt this symmetry as per reports for Ga-LLZO (see ESI $\uparrow$ for narrow $2 \theta$ range XRDs). I $\overline{4} 3 d$ Ga-LLZO systems are thought to possess a La occupancy $<1$, however attempts to similarly refine the Nd site occupancy for Ga-NLZO yielded an unstable refinement, hence the Nd occupancy was set to 1 . Future work will be needed to ascertain if a fractional occupancy occurs and to what extent. Attempts to reduce the $\mathrm{Ga}$ content and so prepare $\mathrm{Ga}_{0.15} \mathrm{NLZO}$ were unsuccessful giving a mixed cubic/tetragonal phase with low conductivity, see ESI. $\uparrow$ The Al-NLZO sample did not have any additional peaks, and hence was assumed to have Ia $\overline{3} d$ symmetry, as per the report from Howard et al. ${ }^{3}$

The undoped NLZO demonstrated significant peak splitting when compared to the cubic counterparts, consistent with the expected reduction in symmetry from a cubic to a tetragonal cell, hence was indexed on the structural model from Howard et al. (space group $I 4_{1} /$ acd) giving similar lattice parameters, see Table 1.

\section{SEM (EDX)}

Fig. 3 displays SEM images and EDX maps of Ga-NLZO powders. Images illustrate similar grain characteristics throughout with no discernible changes in contrast across individual grains, indicating single phase materials. EDX maps show a uniform distribution of the expected elements across the analysed grains, thus confirming elemental composition and a lack of $\mathrm{Al}$ contamination from the crucible. Fig. 4 also shows the surface of a Ga-NLZO pellet, showing closely packed grains, indicating high density materials, and similar EDX results as the corresponding powder.

\section{Conductivity measurements}

The conductivities of the $\mathrm{Nd}$ garnet systems were shown, in general, to increase in line with increased $\mathrm{Li}$ content and larger lattice parameters. A typical Nyquist plot with Au blocking electrodes is shown in Fig. 5; these were fit to a single R/ CPE component in parallel, illustrative of overlapping bulk and grain boundary contributions. Capacitance values and

Table 1 Lattice parameters, relative density, conductivity, and activation energy of $\mathrm{Li}_{5+x} \mathrm{Nd}_{3} \mathrm{Nb}_{2-x} \mathrm{Zr}_{x} \mathrm{O}_{12}(0 \leq x \geq 2)$, Ga/Al-NLZO and NLZO. GaNLZO* refer to the second pellet ran in a Swagelok cell

\begin{tabular}{llclll}
\hline Sample $(x=)$ & Lattice parameters $(a)(\AA)$ & Lattice parameters $(c)(\AA)$ & $\rho_{\text {rel }}(\%)$ & $\sigma_{\text {total }}\left(\mathrm{S} \mathrm{cm}^{-1}\right)\left(50{ }^{\circ} \mathrm{C}\right)$ & Activation energy $(\mathrm{eV})\left(20-143{ }^{\circ} \mathrm{C}\right)$ \\
\hline 0 & $12.6100(1)$ & 71 & $6.1 \times 10^{-6}$ & 0.53 \\
0.5 & $12.6587(5)$ & 69 & $6.9 \times 10^{-6}$ & 0.40 \\
0.75 & $12.6791(2)$ & 74 & $2.0 \times 10^{-5}$ & 0.41 \\
1.2 & $12.7282(1)$ & 91 & $1.4 \times 10^{-4}$ & 0.38 \\
1.4 & $12.7437(1)$ & $12.5503(6)$ & 78 & $1.2 \times 10^{-4}$ & 0.41 \\
2 & $12.9221(7)$ & 78 & $6.2 \times 10^{-7}$ & 0.48 \\
$\mathrm{Al}_{0.2}$ & $12.7994(7)$ & 88 & $3.1 \times 10^{-6}$ & 0.39 \\
Ga-NLZO $_{\text {Ga-NLZO }}^{*}$ & $12.7945(1)$ & 93 & $6.6 \times 10^{-4}$ & 0.25 \\
& $12.7945(1)$ & & & $0.32\left(18-73{ }^{\circ} \mathrm{C}\right)$
\end{tabular}




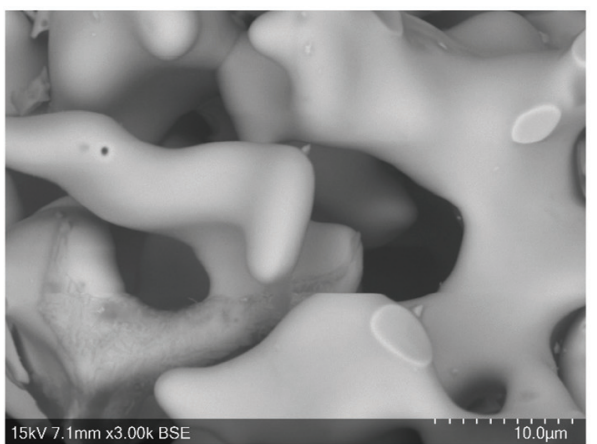

$\mathrm{Ga} L \alpha 1,2$

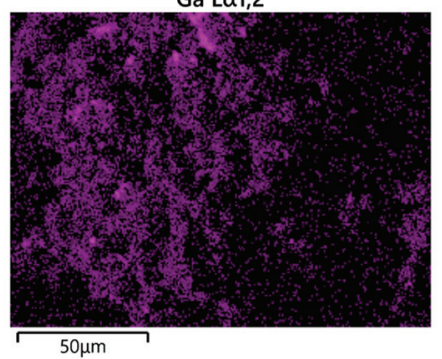

$\mathrm{O} K \alpha 1$

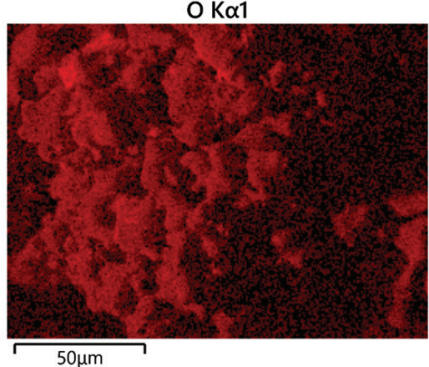

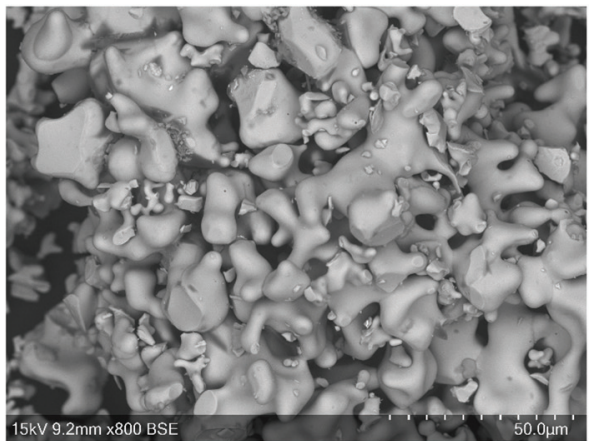

Nd L $\alpha 1$

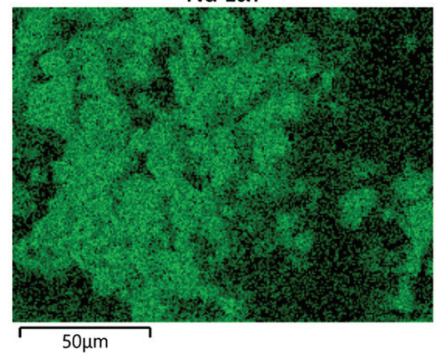

$\operatorname{Zr} L \alpha 1$

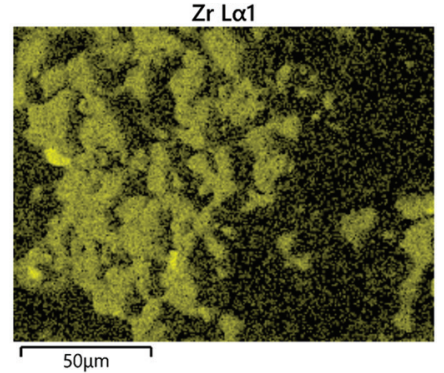

Fig. 3 SEM image and EDX map of elemental distribution in $\mathrm{Ga}_{0.2} \mathrm{Li}_{6.4} \mathrm{Nd}_{3} \mathrm{Zr}_{2} \mathrm{O}_{12}$ in powder form.

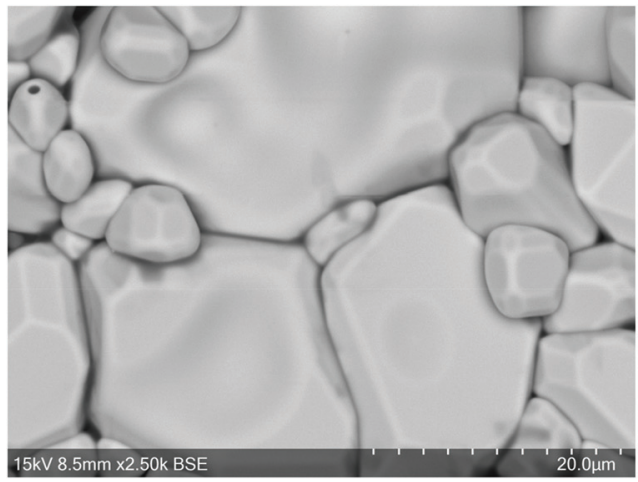

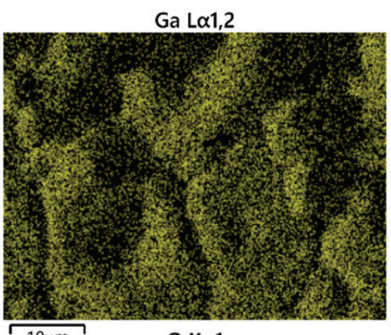

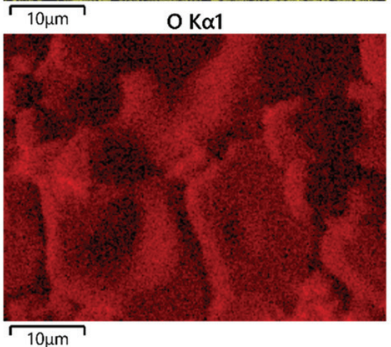

$\mathrm{Nd} \operatorname{L\alpha 1}$

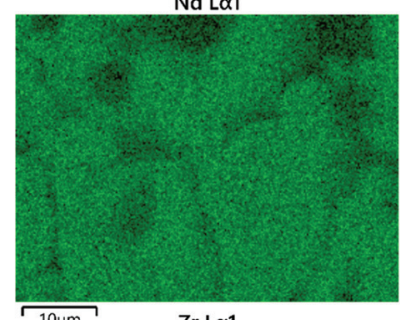

$\operatorname{ZrL} \alpha 1$

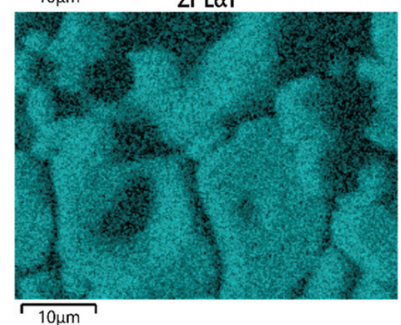

Fig. 4 SEM image and EDX map of elemental distribution in from the pellet surface of $\mathrm{Ga}_{0.2} \mathrm{Li}_{6.4} \mathrm{Nd}_{3} \mathrm{Zr}_{2} \mathrm{O}_{12}$.

dielectric constants fall within the range expected for bulk materials, see ESI. $\dagger$ The measurements for Ga-NLZO at $\sim 20{ }^{\circ} \mathrm{C}$ displayed a buried contribution in the double layer capaci- tance spike relating to the Au electrodes, perhaps relating to a small grain boundary component. However, this was unable to be fitted to the corresponding R/CPE element, see Fig. 5 inlay. 


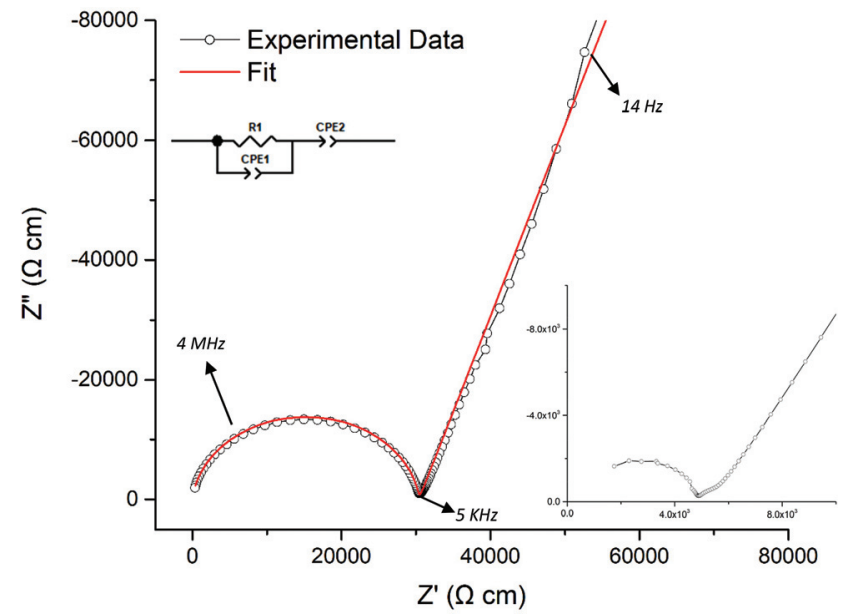

Fig. 5 Typical Nyquist plot of $\mathrm{Li}_{5.75} \mathrm{Nd}_{3} \mathrm{Nb}_{1.25} \mathrm{Zr}_{0.75} \mathrm{O}_{12}$ at $63^{\circ} \mathrm{C}$ with $\mathrm{Au}$ blocking electrodes. The plots were fit to a single R/CPE component, illustrative of overlapping bulk and grain boundary contributions. More conductive plots, such as those in Fig. 8, required the addition of an inductive element. Inlay is Ga-NLZO at $18{ }^{\circ} \mathrm{C}$, demonstrating buried grain boundary contribution in the double layer capacitance spike.

Highly conductive samples, such as Ga-NLZO, demonstrated a high frequency inductance $\left(\sim 10^{-6} \mathrm{H}\right)$, hence an additional inductive element was included in the equivalent circuit model, see Fig. 8. This corresponds to previous reports and likely arises from the system wiring and/or limitations of the impedance equipment. ${ }^{34,68,81}$

The conductivity of $\mathrm{Li}_{5+{ }_{x}} \mathrm{Nd}_{3} \mathrm{Nb}_{2-x} \mathrm{Zr}_{x} \mathrm{O}_{12}$ garnets reached a maximum of $7 \times 10^{-5} \mathrm{~S} \mathrm{~cm}^{-1}\left(22{ }^{\circ} \mathrm{C}\right)$ for $\mathrm{Li}_{6.2} \mathrm{Nd}_{3} \mathrm{Zr}_{1.2} \mathrm{Nb}_{0.8} \mathrm{O}_{12}$ before falling noticeably for $\mathrm{Li}_{6.4} \mathrm{Nd}_{3} \mathrm{Zr}_{1.4} \mathrm{Nb}_{0.6} \mathrm{O}_{12}$ attributed to decreased densification of the latter. The conductivity of AlNLZO was considerably lower at $6 \times 10^{-6} \mathrm{~S} \mathrm{~cm}^{-1}\left(22{ }^{\circ} \mathrm{C}\right)$, however Ga-NLZO showed a much higher conductivity at $2 \times$ $10^{-4} \mathrm{~S} \mathrm{~cm}^{-1}\left(20^{\circ} \mathrm{C}\right)$, and was the only $\mathrm{Nd}$ based garnet to demonstrate room temperature conductivity in the $10^{-4} \mathrm{~S}$ $\mathrm{cm}^{-1}$ range.

Arrhenius plots, see Fig. 6 and 7, demonstrate a mostly linear trend, with activation energies given in Table 1. NLZO shows some deviation from linearity which is attributed to water loss, as a result of proton-Li exchange. However, non-linearity is present for the high $\mathrm{Li}$ content cubic phase $\mathrm{Nd}$ garnets between the room temperature measurements and $50{ }^{\circ} \mathrm{C}$, which cannot be explained similarly.

As the room temperature measurements, particularly in the high content $\mathrm{Li} \mathrm{Nd}$ garnets, were Au sputtered and $50{ }^{\circ} \mathrm{C}$ measurements were taken post quenching while curing the $\mathrm{Au}$ paste, it can be concluded that the heating step to cure the Au paste (and/or subsequent quenching from $>700{ }^{\circ} \mathrm{C}$ ) may be responsible. Comparative impedance spectra analysis of pre/ post quenched Ga-NLZO pellets revealed a newly resolved grain boundary contribution post quenching, which fits to two parallel R/CPE components connected in series rather than one, see Fig. 8. This additional grain boundary contribution reduced the conductivity, with this being so even if the buried

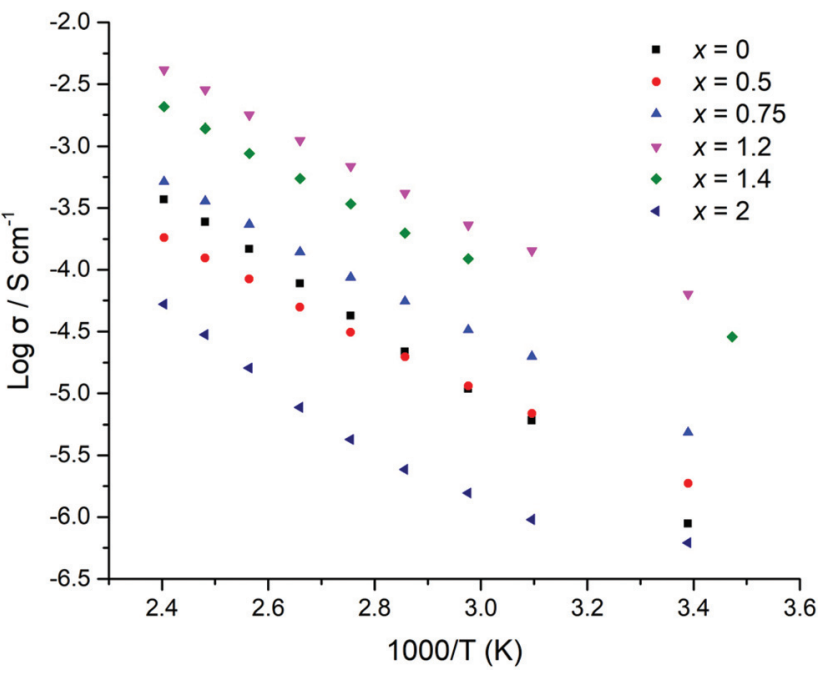

Fig. 6 Arrhenius plots of $\mathrm{Li}_{5+x} \mathrm{Nd}_{3} \mathrm{Nb}_{2-x} \mathrm{Zr}_{x} \mathrm{O}_{12}(0 \leq x \geq 2)$. The non-linearity observed is a result of quenching degrading the microstructure. The non-linearity of the tetragonal sample is attributable to water loss, as although samples were stored in a Ar glovebox, it was not possible characterise fully under Ar. The lower temperatures measurement for $\mathrm{Li}_{6.4} \mathrm{Nd}_{3} \mathrm{Nb}_{0.6} \mathrm{Zr}_{1.4} \mathrm{O}_{12}$ is due to lower temperature experienced in the Lab. Note $-x=0-0.75$ were not Au sputtered first as conductivity was expected to be low, hence linear trend.

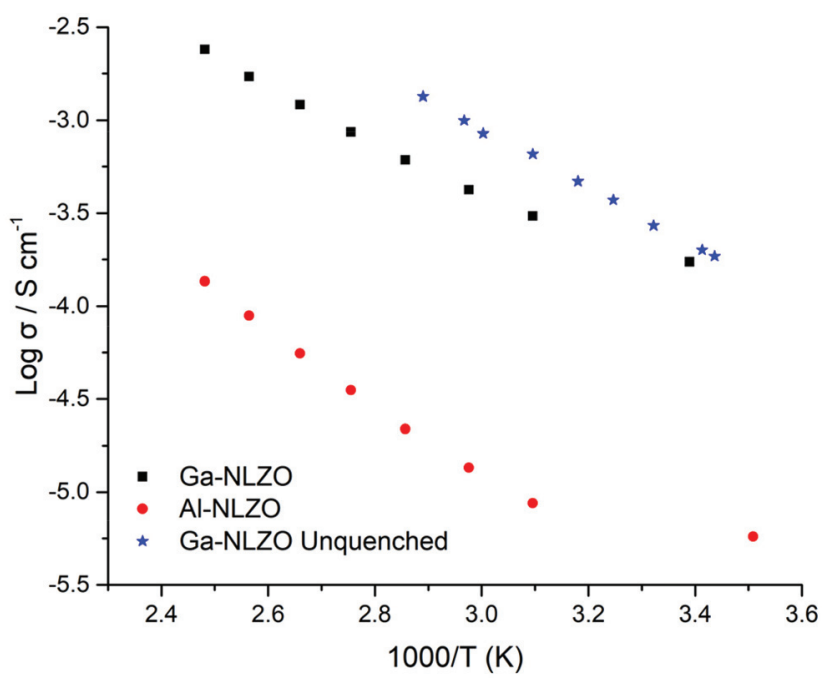

Fig. 7 Arrhenius plots of $\mathrm{Al}_{0.2} \mathrm{Li}_{6.4} \mathrm{Nd}_{3} \mathrm{Zr}_{2} \mathrm{O}_{12}$ and $\mathrm{Ga}_{0.2} \mathrm{Li}_{6.4} \mathrm{Nd}_{3} \mathrm{Zr}_{2} \mathrm{O}_{12}$. As bending of the plots were noted between room temperature and $50{ }^{\circ} \mathrm{C}$, a fresh $\mathrm{Ga}_{0.2} \mathrm{Li}_{6.4} \mathrm{Nd}_{3} \mathrm{Zr}_{2} \mathrm{O}_{12}$ pellet was $\mathrm{Au}$ sputtered, secured in a Swagelok cell and measured from $18-73^{\circ} \mathrm{C}$ (blue stars), gave a linear plot and confirmed the degradation encountered with $\mathrm{Nd}$ garnets when subjected to quenching.

contribution in Fig. 5 inlay is considered. As Ga-NLZO is the focus of this work, a pellet was sputtered with $\mathrm{Au}$, cooled naturally and analysed further between $18-73{ }^{\circ} \mathrm{C}$. The Arrhenius plots demonstrate near perfect linearity, thus indicating quenching negatively affects the conductivity of $\mathrm{Nd}$ based garnets, see Fig. 7. 

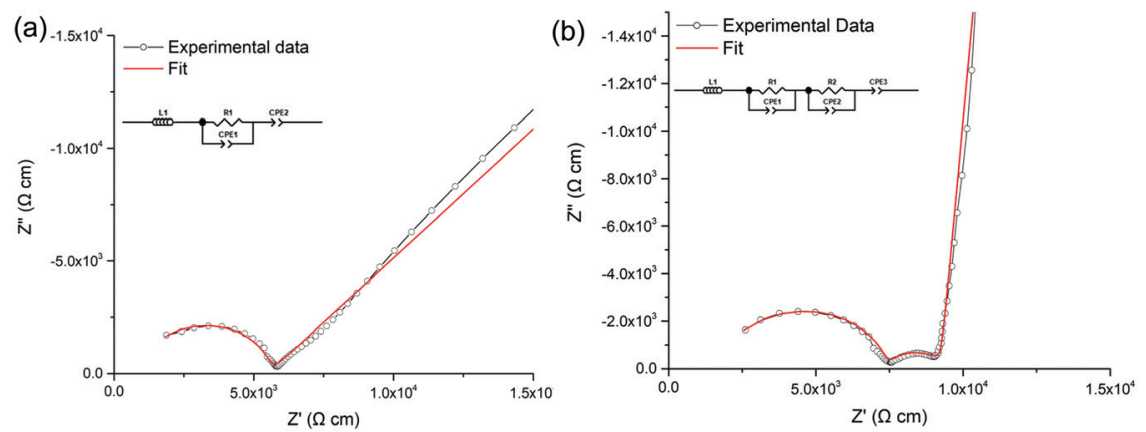

Fig. 8 Nyquist plot of $\mathrm{Ga}_{0.2} \mathrm{Li}_{6.4} \mathrm{Nd}_{3} \mathrm{Zr}_{2} \mathrm{O}_{12}$ at $22{ }^{\circ} \mathrm{C}$ where (a) is Au sputtered and fit to a single R/CPE component and (b) is the post quenched plot of the same sample after drying of Au paste, now showing a grain boundary contribution and fitting to two parallel R/CPE components connected in series. The new features in b, which lower total system conductivity, relate to degradation of the pellet microstructure from quenching. This is suspected to relate to thermal shock and shows that $\mathrm{Nd}$ based garnets respond less favourably to quenching than other lithium garnets, whereupon increased conductivity is commonly observed.

In order to try to evaluate the origin of this detrimental effect of quenching, fresh Ga-NLZO pellets were quenched for microstructural evaluation, however these violently shattered indicating thermal shock. Hence the results suggest that Nd garnets are degraded by quenching, although more work is needed to confirm the origin of this affect. In contrast, a furnace cooled Ga-NLZO pellet demonstrated an improved $93 \%$ relative density and ionic conductivity of $0.3 \mathrm{mS} \mathrm{cm}{ }^{-1}$ at $20{ }^{\circ} \mathrm{C},\left(1.34 \mathrm{mS} \mathrm{cm}^{-1}\right.$ at $\left.73{ }^{\circ} \mathrm{C}\right)$. See Table 1 .

Overall the evaluation of $\mathrm{Nd}$ based garnets has revealed that, despite many having rather low room temperature conductivity, the conductivity of Ga-NLZO is similar to other lithium garnets prepared under similar conditions, despite the smaller lattice parameters. ${ }^{34,42,50,52,55,82-85}$ The high conductivity observed for Ga-NLZO is likely due, in part, to the good sinterability of this composition leading to higher density (93\% theoretical).

The high-density of Ga-NLZO pellets (93\%) is notable as it is accomplished relatively simply, and are higher than our previous reports for La based garnets prepared under similar conditions, where densities $\leq 90 \%$ are more commonly observed. ${ }^{32,53,54,86}$

\section{Cell testing - interfacial resistance}

To assess the ASR in contact with Li metal, a sintered GaNLZO pellet was assembled into Li metal symmetric cells. $\mathrm{Li}$ stripping and plating was then investigated to assess cycling and ASR stability (see later). Cells were analysed via impedance spectroscopy prior to (and after) cell testing. A typical Nyquist impedance plot is shown in Fig. 9. The impedance spectrum was fit to three parallel R/CPE components, with the bulk and grain boundary contribution being present in the high to midfrequency range, which is now more resolved than the data from the earlier conductivity measurements (Fig. 5 inlay). The conductivity values obtained from this plot are in good agreement with those values obtained prior $\left(\sim 2.5 \times 10^{-4} \mathrm{~S} \mathrm{~cm}^{-1}\right.$, all pellets $\sim 93 \%$ density). Several pellets were analysed, which demonstrated similar characteristics. The semi-circle observed

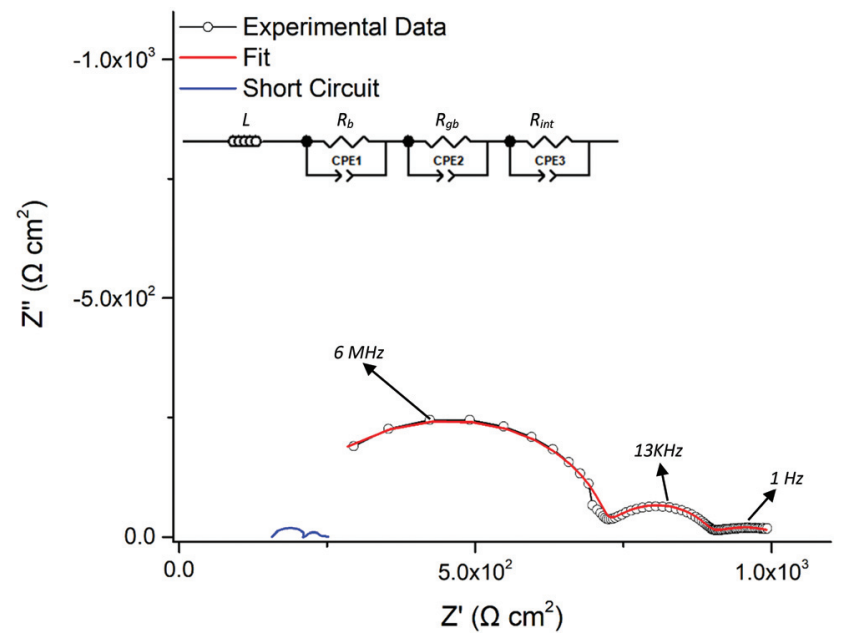

Fig. 9 Li symmetry cell Nyquist plot of $\mathrm{Ga}_{0.2} \mathrm{Li}_{6.4} \mathrm{Nd}_{3} \mathrm{Zr}_{2} \mathrm{O}_{12}$, which was fit to three R/CPE components connect in series, illustrative of bulk, grain and Li/garnet interface contributions. The absence of $\mathrm{Au}$ has revealed the entire grain boundary contribution, previously thought buried in the charging spike. Blue line is representative of a cell after short circuit.

at low frequency in Fig. 9 corresponds to the resistance encountered at the $\mathrm{Li}$ metal/garnet interface. The area specific resistance (ASR) is hence determined from this semi-circle.

As a wide variety of potential Ga-Li eutectics can be formed from room temperature to $175{ }^{\circ} \mathrm{C}$, impedance analysis was undertaken after hand pressing the Li foils only $\left(20^{\circ} \mathrm{C}\right)$, using the $\mathrm{G}$ clamp pressure $\left(20^{\circ} \mathrm{C}\right)$ and at different temperatures under the clamp pressure $\left(60-175^{\circ} \mathrm{C}\right)$, with ASR values being taken after pressing and cooling to room temperature. These results are shown in Fig. 10a. Hand pressing the Li foil yielded relatively large ASR values, however these were lower than expected at $4175 \Omega \mathrm{cm}^{2}$ (typically values of $\sim 10000 \Omega \mathrm{cm}^{2}$ are found for other garnets analysed similarly). ${ }^{26,68,87}$ This fell considerably by applying the force of the clamp but remained quite high at $865 \Omega \mathrm{cm}^{2}$. Increased temperature reduced the 
a)
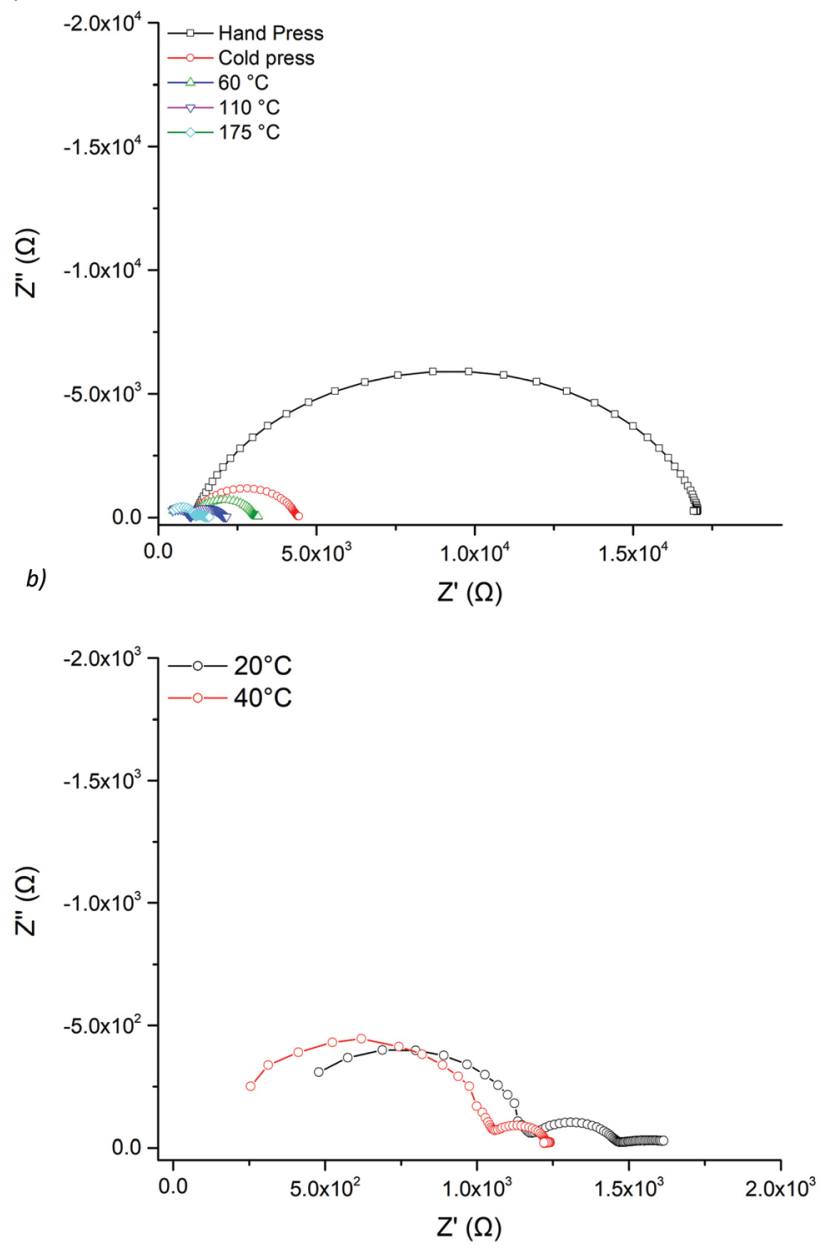

Fig. 10 (a) Impedance spectra demonstrating ASR dependence on heat with the interfacial resistances of 4175 (black, hand pressed), 865 (red, clamp pressure only), 518 (green, $60^{\circ} \mathrm{C}$ ), 274 (blue $\left(110^{\circ} \mathrm{C}\right.$ ) and 128 (teal, $175^{\circ} \mathrm{C}$ ) $\Omega \mathrm{cm}^{2}$. (b) Impedance spectroscopy of Li/Ga-NLZO/Li symmetry cell at room temperature and $40^{\circ} \mathrm{C}$, showing nearly complete removal the $\mathrm{Li} /$ garnet interface contribution, indicating minimal interfacial resistance at slightly elevated temperature. The ASR at room temperature was $67 \Omega \mathrm{cm}^{2}$, and was the lowest recorded in this work. The pellet in (b) had decreased density (91\%), hence has also revealed the grain boundary component more clearly.

ASR further, with the ASR after pressing at $110^{\circ} \mathrm{C}$ being $274 \Omega$ $\mathrm{cm}^{2}$ (at $20^{\circ} \mathrm{C}$ ), which is in the range of many garnet reports which use complex techniques and/or additional coatings. However, heating to $175{ }^{\circ} \mathrm{C}$ was required to enable the lowest possible ASR for Ga-NLZO at $67 \Omega \mathrm{cm}^{2}$, with cells commonly being in the $130-80 \Omega \mathrm{cm}^{2}$ range due to limitations of the 3-way G-clamp. To confirm the low ASR is due to the presence of Ga, a tetragonal NLZO symmetry cell was assembled and pressed in the same manner. This cell demonstrated a high ASR ( 1000 $\Omega \mathrm{cm}^{2}$, see ESI $\dagger$ ). Therefore, this result supports the conclusion that the relatively low ASR encountered with Ga-NLZO arises from the Ga dopant, with results indicating additional reactions occur outside of enhanced $\mathrm{Li}$ metal plastic deformation at increased temperature. Therefore, all cells were heated to $175{ }^{\circ} \mathrm{C}$ under pressure from the G-clamp and left to cool before removing. Impedance was subsequently further analysed on a Li/Ga-NLZO/Li cell at 20 and $40{ }^{\circ} \mathrm{C}$, with the slightly elevated temperature showing almost complete removal of the Li/Ga-NLZO contribution, hence indicating nominal resistance at the interface. See Fig. $10 \mathrm{~b}$.

Ga-NLZO cells thus exhibited much decreased ASR values when compared to the Ga-LLHO, Ce-doped LLZO and Cedoped $\mathrm{Li}_{5+x} \mathrm{La}_{3} \mathrm{Nb}_{2-x} \mathrm{Ce}_{x} \mathrm{O}_{12}$ cells we reported earlier (ASR values $\left.\sim 400 \Omega \mathrm{cm}^{2}\right) .{ }^{32,47,54}$ As cells were pressed in a similar manner, this is a considerable reduction in ASR which can only be attributed to an interfacial reaction between Li metal and Ga-NLZO.

Furthermore, these ASR values are lower than those reported for several LLZO based samples processed fully under Ar in a glove box environment. For example, work by Brugge et al. (and follow up work by Pesci et al.) determined that thermal etching of Ga-LLZO, under controlled conditions (e.g. glovebox polishing and transferring via vacuum for heating), can reduce ASR values from $\sim 12372.2$ to $148.9 \Omega \mathrm{cm}^{2}$, with the main difference being $\mathrm{Li}$ foils were cold pressed on to the garnet surface and left for $24 \mathrm{~h}$ rather than heating to $175^{\circ} \mathrm{C}$ for $1 \mathrm{~h}$. This etched value is still higher than that achieved by Ga-NLZO (67 $\left.\Omega \mathrm{cm}^{2}\right)$ and does not require glovebox connected furnaces. ${ }^{55,68,81}$ Work by Tsai et al. using similar cell making procedures, but employing Au sputtered buffer layers between garnet and Li foil, were also shown to reduce interfacial resistance, however were still significantly higher than Ga-NLZO (380 vs. $67 \Omega \mathrm{cm}^{2}$ ). ${ }^{68,88}$ Many other works also detail interfacial modifications (e.g. polymers, pulsed laser deposition)/complex procedures/expensive alloying reactions (e.g. Au, Ag) which give higher resistances than the as prepared Ga-NLZO cells ${ }^{87,89-100}$ and many others which are similar. ${ }^{87,101-103}$ Hence it can be concluded that this $\mathrm{Nd}$ based garnet does indeed possess intrinsically lower ASR values than other (La based) garnet materials.

These ASR values could, perhaps, be much improved if the material were able to be processed fully in an Ar glovebox or if the pressure could be accurately controlled/increased to much higher values. However, such low resistances intrinsic to lithium garnets are rare without compromising other desirable qualities, and if Ga-NLZO were used as a conductive interface (CI) layer, it could be co sintered with other SSEs (hence not requiring any additional energy intensive techniques), thus considerably reducing costs.

\section{Cyclic voltammetry}

Before analysing the interfacial stability of Ga-NLZO, the electrochemical stability window was assessed via cyclic voltammetry, see Fig. 11. Peaks are present at $\sim 0.3 \mathrm{~V}$ and $\sim-0.4$ which indicate $\mathrm{Li}$ stripping and $\mathrm{Li}$ plating respectively, the small peaks $\sim 1-1.5 \mathrm{~V}$ is attributed to Li/Au alloying. ${ }^{34}$ Outside of this region, the peak profile remains flat with an absence of redox peaks, thus confirming the stability up to $5 \mathrm{~V}\left(v s . \mathrm{Li} / \mathrm{Li}^{+}\right)$. A negligible current response is present throughout the rest of the voltammogram, indicating low electronic conductivity. 


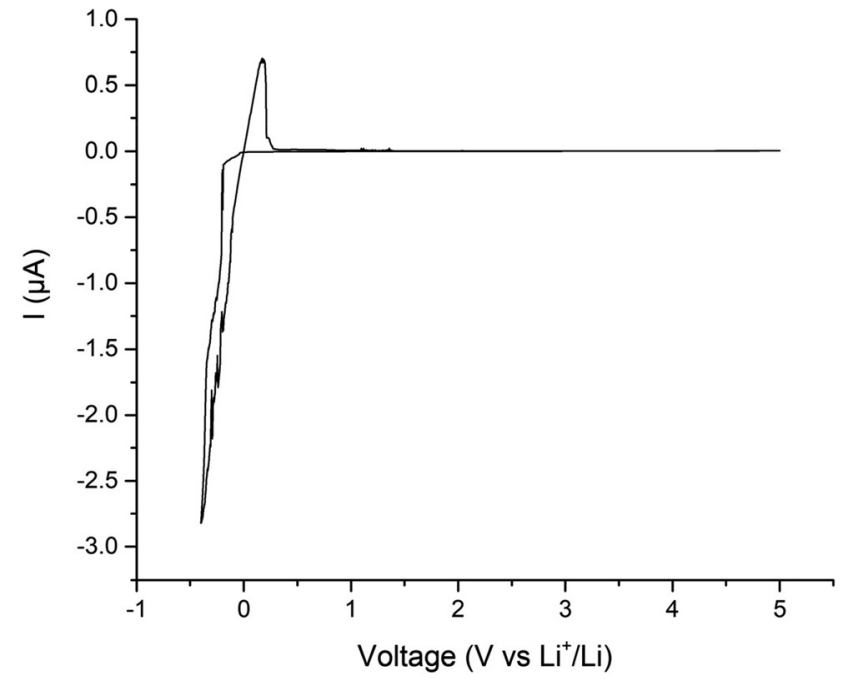

Fig. 11 Cyclic voltammetry between -0.4 to $5 \mathrm{~V}$ vs. $\mathrm{Li}_{+} / \mathrm{Li}$ of Ga-NLZO at $1 \mathrm{mV} \mathrm{s}^{-1}$. Small peaks $\sim 1-1.5 \mathrm{~V}$ are attributed to Au/Li alloying.

\section{Cell testing - Li stripping and plating}

Li stripping/plating were investigated for the assembled symmetric cells to assess cycling and ASR stability. The cell was left on an open lab bench to approximate real world use, hence small temperature variations were present (between 15-21 ${ }^{\circ} \mathrm{C}$ ). The results showed that Ga-NLZO exhibits excellent cycling stability at room temperature at 12.5 and $25 \mu \mathrm{A} \mathrm{cm}^{-2}$, see Fig. 12. The small fluctuations in the voltage profile relate to the slight alterations in the temperature, with slightly higher voltages occurring overnight and lower voltages during the day. To confirm the voltage stability, a cell was cycled at $50{ }^{\circ} \mathrm{C}$, which shows mostly flat voltage profiles for $\sim 83$ hours. Therefore, no interface degradation is apparent and voltage fluctuations are due to real life temperature variations. However, a large voltage drop is present in Fig. 12 when the current density is increased to $50 \mu \mathrm{A} \mathrm{cm}{ }^{-2}$, which indicates a short circuit due to dendrite propagation. This dendrite propagation at low current densities suggest that the reaction between $\mathrm{Li}$ and the $\mathrm{Ga}$ dopant must propagate through the pellet.

To confirm the short circuit, critical current density (CCD) analysis was conducted on a fresh cell (where CCD is defined as the maximum current which can be applied to a cell before dendritic lithium propagation and short circuiting occurs). CCDs of the lithium garnet systems are thought to depend primarily upon the pellet density (which should be as high as possible), grain boundary characteristics, conductivity, and the ASR (which should be as low as possible). ${ }^{26,31,55,104-106}$ ASR for this cell was calculated at $95 \Omega \mathrm{cm}^{2}$, pellet density at $\sim 90 \%$ and conductivity in the $0.2 \mathrm{mS} \mathrm{cm}^{-1}$ range. The cell was left on an open lab bench where temperature fluctuated between 20-22 ${ }^{\circ} \mathrm{C}$. As shown in Fig. 13, the cell follows ohmic currentvoltage behaviour up to $50 \mu \mathrm{A} \mathrm{cm} \mathrm{cm}^{-2}$, at which point a small voltage drop is noted indicative of a short circuit. The short a)

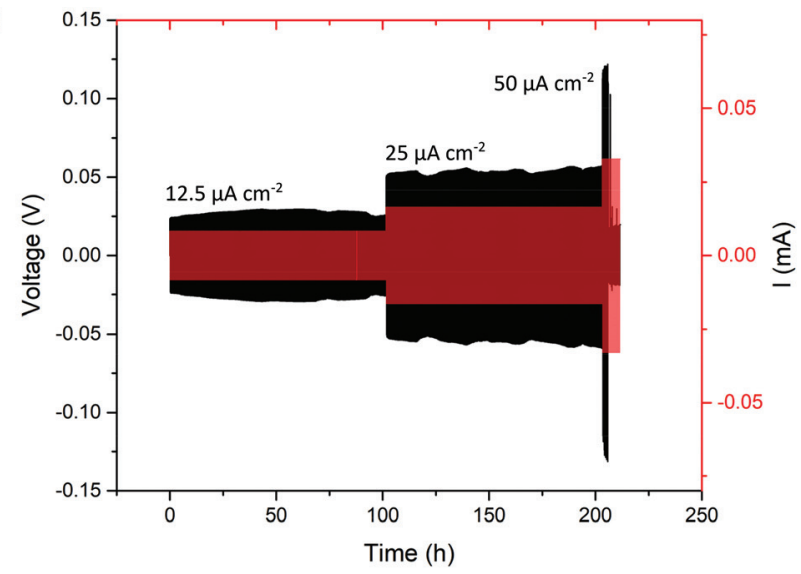

b)

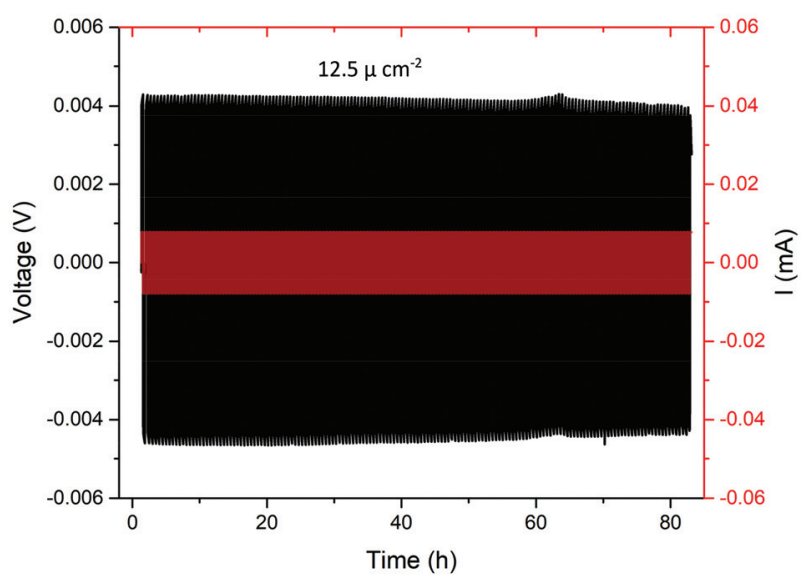

Fig. 12 Lithium stripping and plating of Li/Ga-NLZO/Li cell (a) at different current densities at room temperature on open lab bench (between $15-21^{\circ} \mathrm{C}$ throughout the day) and (b) at $50{ }^{\circ} \mathrm{C}$ to demonstrate flat voltage profile at controlled temperature.

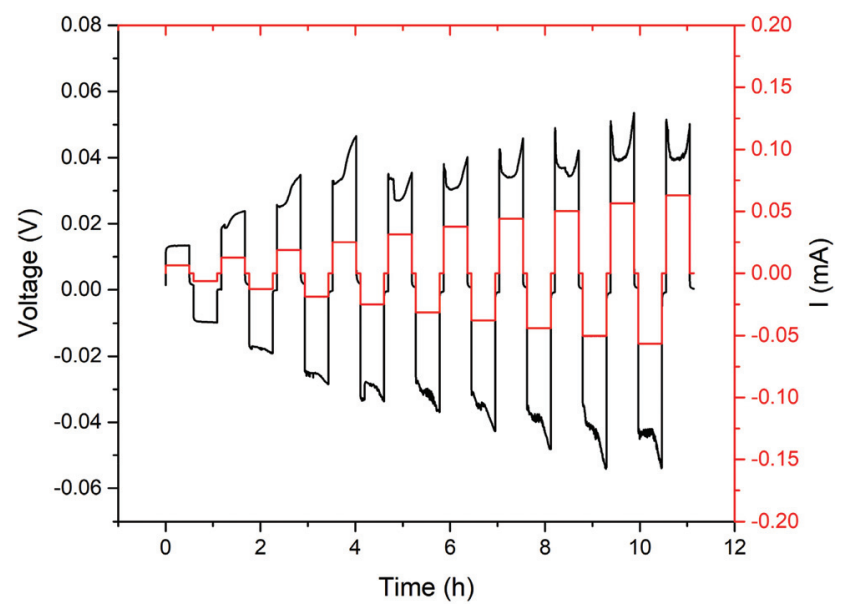

Fig. 13 Critical current density analysis, starting at $10 \mu \mathrm{A} \mathrm{cm}{ }^{-2}$, and increasing in increments of $10 \mu \mathrm{A} \mathrm{cm}^{-2}$. A small voltage drop is noted at $50 \mu \mathrm{A} \mathrm{cm}{ }^{-2}$ which corresponded to short circuit. This was confirmed via impedance spectroscopy and was analogous the impedance spectrum found in Fig. 9. 
circuit was confirmed by impedance spectroscopy, giving a similar Nyquist plot to Fig. 9. Efforts were made to improve this value by ball milling Ga-NLZO powders for $10 \mathrm{~h}$ to improve particle size distribution post sintering. This gave the same CCD value. This is indicative of the processes involved in the low ASR values, which we attribute to the formation of a Ga-Li eutectic at the grain boundary, propagating through the SSE during Li stripping and plating. This, therefore suggests that Ga-NLZO cannot be utilized as an electrolyte on its own with Li metal anodes, but may find use as a CI layer in conjunction with another garnet electrolyte.

Therefore, a proof-of-concept Li/Ga-NLZO/Ta-LLZCO/Ga$\mathrm{NLZO/Li}$ layered cell was assembled and can be found in Fig. 14. This pellet was prepared and densified via a similar procedure, which resulted in a dense pellet with fused interfaces. The pellet was then analysed via impedance spectroscopy with $\mathrm{Au}$ electrodes and assembled into symmetric cells, with the latter demonstrating no mechanical interfacial issues while sanding to a mirror finish, see Fig. 14. All Impedance analysis yielded no additional interface contributions, hence indicating well sintered and connected interfaces. This symmetric cell demonstrated a low ASR value of $102 \Omega \mathrm{cm}^{2}$, similar to Ga-NLZO on its own, with a conductivity at $21{ }^{\circ} \mathrm{C}$ of $2.5 \times 10^{-4} \mathrm{~S} \mathrm{~cm}^{-1}$. The layered cells showed extended cycling stability, over multiple current densities (up to at least $100 \mu \mathrm{A} \mathrm{cm}{ }^{-2}, \sim 500 \mathrm{~h}$ ), significantly beyond the CCD noted solely for the Ga-NLZO garnets. The large voltage drop (indicative of a short circuit) seen for the Ga-NLZO materials in Fig. 12 is absent from the layered cell data, indicating no short circuit over the analysed current densities. Hence GaNLZO can be readily co-sintered with other garnets as an interface layer to increase the CCD while maintaining the low ASR values.

\section{Post cycling analysis}

In order to gain more information on the short circuited GaNLZO cells, a short-circuited symmetric cell was disassembled, and Li metal removed by melting to reveal the pellet surface, which were subsequently analysed by SEM/EDX and compared with a freshly prepared Ga-NLZO pellet. As the polishing procedure does not yield a surface from which SEM/EDX was able to distinguish any unusual features (see ESI $\dagger$ ), the shortcircuited pellet was thermally etched via the densification procedure used for fresh $\mathrm{Nd}$ garnet pellets, with the aim of also regenerating the pellet. After this heat treatment, individual grains were well resolved (no mother powder was used). The SEM images of the pellet surface revealed unusual elemental clusters, as distinguished from differences in contrast from the BSE image, with grain boundaries showing clear changes in morphology, see Fig. 15. These features were largely present across the entire pellet surface, but were only sparingly present on fresh pellets prior to cycling, and hence they have dramatically increased post Li metal adhesion and cycling. EDX of these clusters revealed accumulations of $\mathrm{Zr}$, encased by $\mathrm{Nd}$ surround by Ga plated grain boundaries, see Fig. 15. This yields evidence of $\mathrm{Ga}$ exsolution on the pellet surface, which
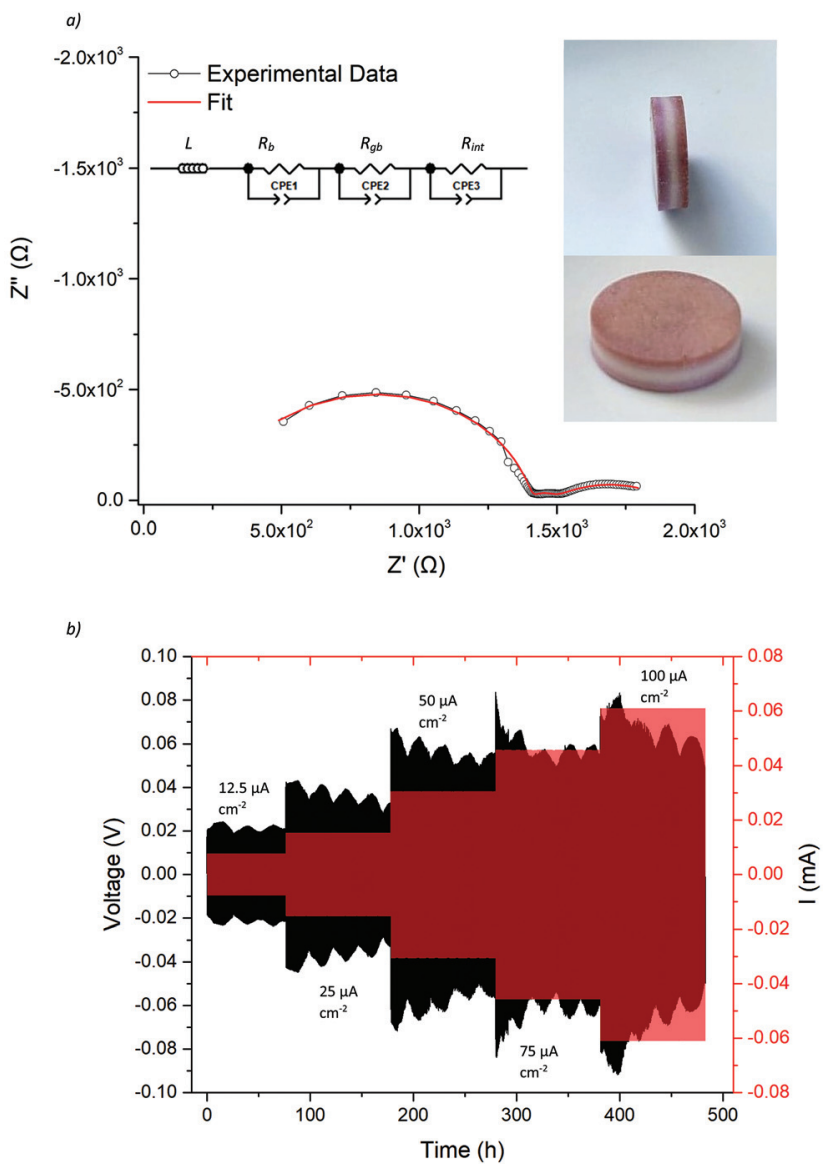

c)

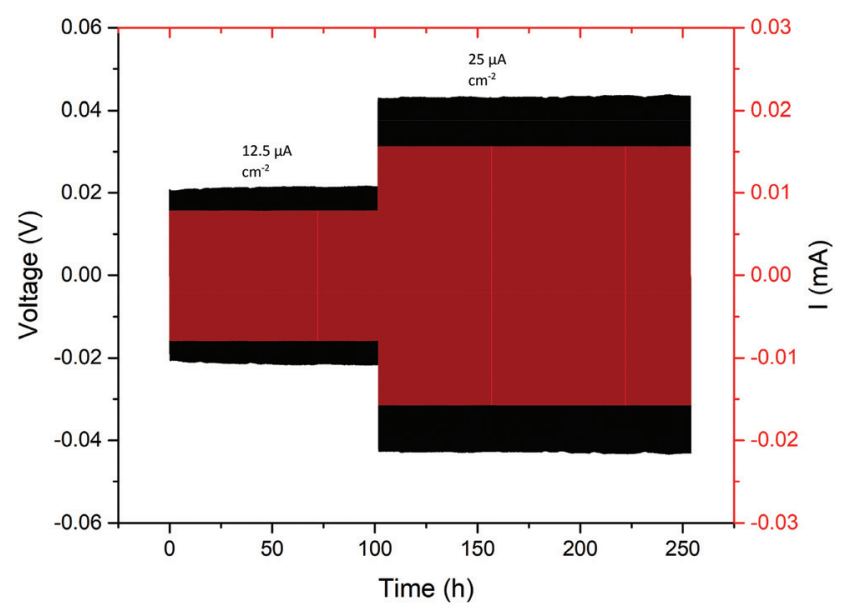

Fig. $14 \mathrm{Li} / \mathrm{Ga}-\mathrm{NLZO} / \mathrm{Ta}-\mathrm{LLZCO} / \mathrm{Ga}-\mathrm{NLZO} / \mathrm{Li}$ sandwich cells, where (a) is the impedance spectrum of a symmetry cell, fit to three R/CPE parallel components connected in series. Considering the lack of any additional features in the spectrum only three R/CPE components were used, irrespective of the layered structure. Inlay photographs are of the formed pellet, where purple is Ga-NLZO, and white is Ta-LLZCO. (b) is the Li striping and plating stability of the cell at different current densities. The cell was cycled at room temperature on an open lab bench to approximate real world use during summer $\left(15-27^{\circ} \mathrm{C}\right)$, hence fluctuations in the voltage profile. These are more noticeable compared to Fig. 12 due to larger temperature differential, lower voltage troughs occur overnight, whereas high voltage peaks occur in the day. (c) confirms voltage stability when cycled at a fixed temperature $\left(43^{\circ} \mathrm{C}\right)$. 

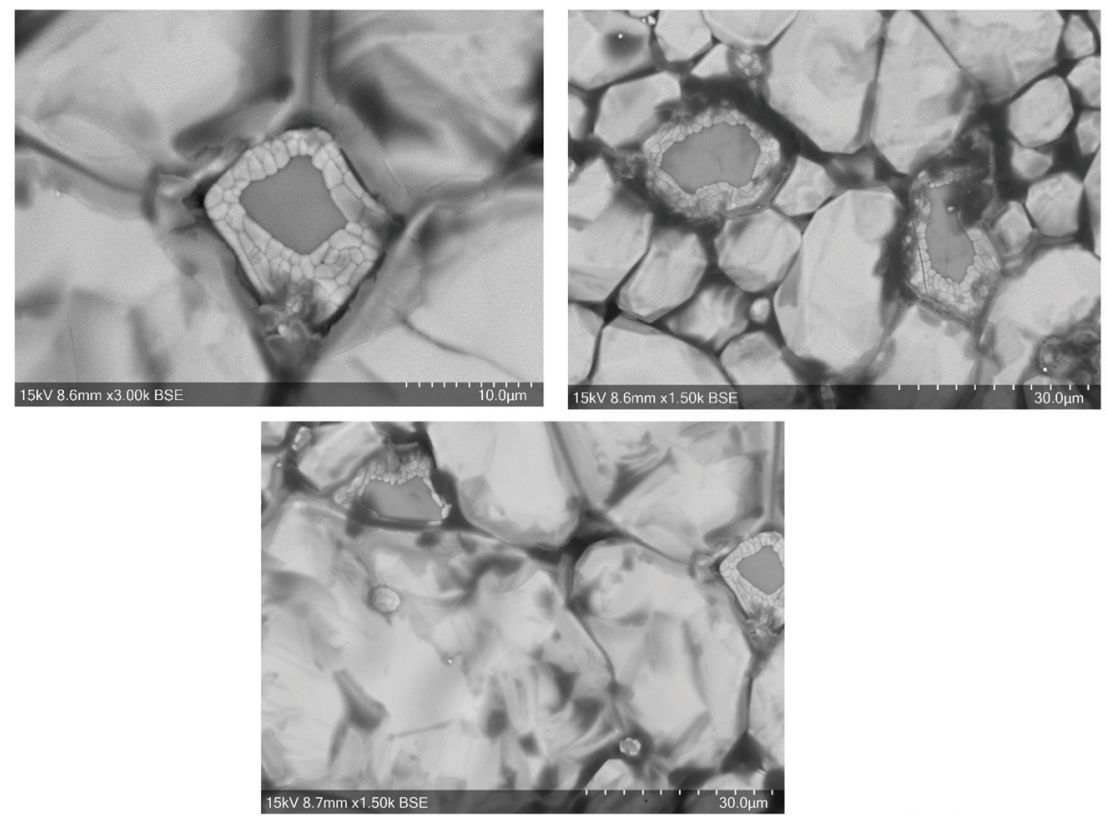

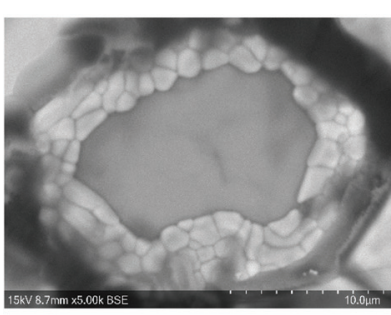

Ga $L \alpha 1,2$
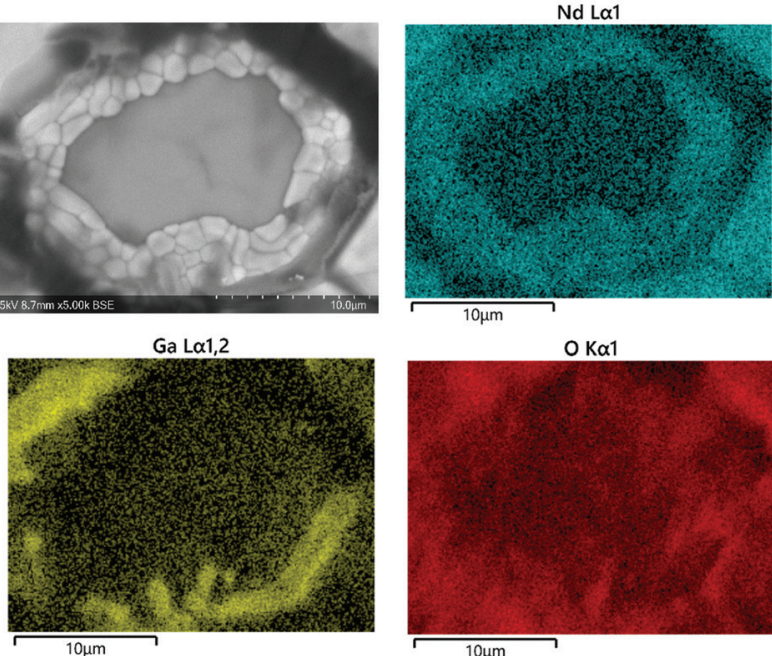

O $K \alpha 1$

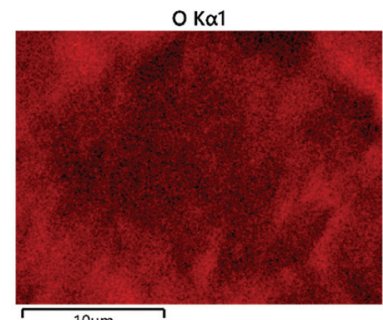

$10 \mu \mathrm{m}$

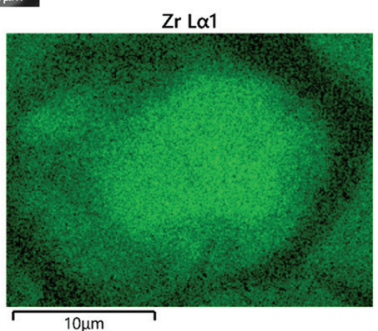

Al Ka1

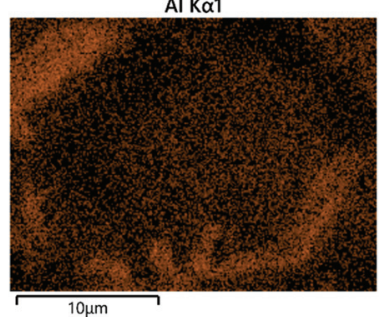

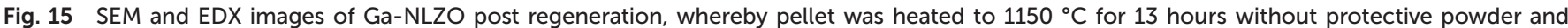

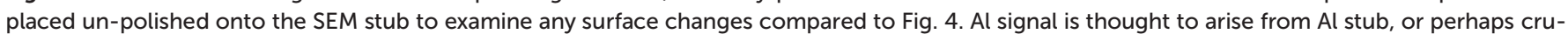
cible related $\mathrm{Al} / \mathrm{Li}$ exchange, as this is the same pellet seen in Fig. 3, where no Al signal was detected.

we attribute as contributing to the low CCD by way of Ga-Li eutectic alloys.

A cross section of the pellet was examined and some areas of altering contrast were seen, particularly in Fig. 16, with EDX demonstrating some corresponding evidence of elemental separation in some areas. This indicates these elemental clusters propagate through the pellet and could be related to the observed low CCD. However, further work is needed to provide confirmation.

As large quantities of these surface impurities were present, the pellet was analysed by XRD which showed that it still appeared to be a mostly pure cubic garnet, see Fig. 17. The pellet surface (pre thermal etching) demonstrated some small background peaks corresponding to Ga-Li eutectics, however these peaks are difficult to resolve due to the weak scattering ability of $\mathrm{Li} / \mathrm{Ga}$ and will require use of $\mathrm{Li}$ sensitive techniques, such as time of flight secondary ion mass spectroscopy, for further confirmation.

To provide some confirmation of our hypothesis concerning $\mathrm{Li}-\mathrm{Ga}$ eutectic formation through $\mathrm{Ga}$ exsolution, we examined the effect of placing $\mathrm{Li}$ metal discs in contact with a $\mathrm{Ga}_{2} \mathrm{O}_{3}$ pellet and heating to $175^{\circ} \mathrm{C}(1 \mathrm{~h})$ and also left a $\mathrm{Ga}_{2} \mathrm{O}_{3}$ pellet in contact with the metal at room temperature for two days. XRD analysis was subsequently undertaken under inert conditions, with freshly scratched Li metal also analysed for comparison. Both cases indicated a clear reaction with $\mathrm{Li}$ metal, with the heated sample fully decomposing Li metal into a black powder. In the heated sample, phases were matched to both $\mathrm{Li}_{2} \mathrm{Ga}$ and $\mathrm{Li}-\mathrm{Ga}$ eutectics (see ESI $\dagger$ ). Comparing these data to the XRD of the Ga-NLZO pellet surface shows corres- 

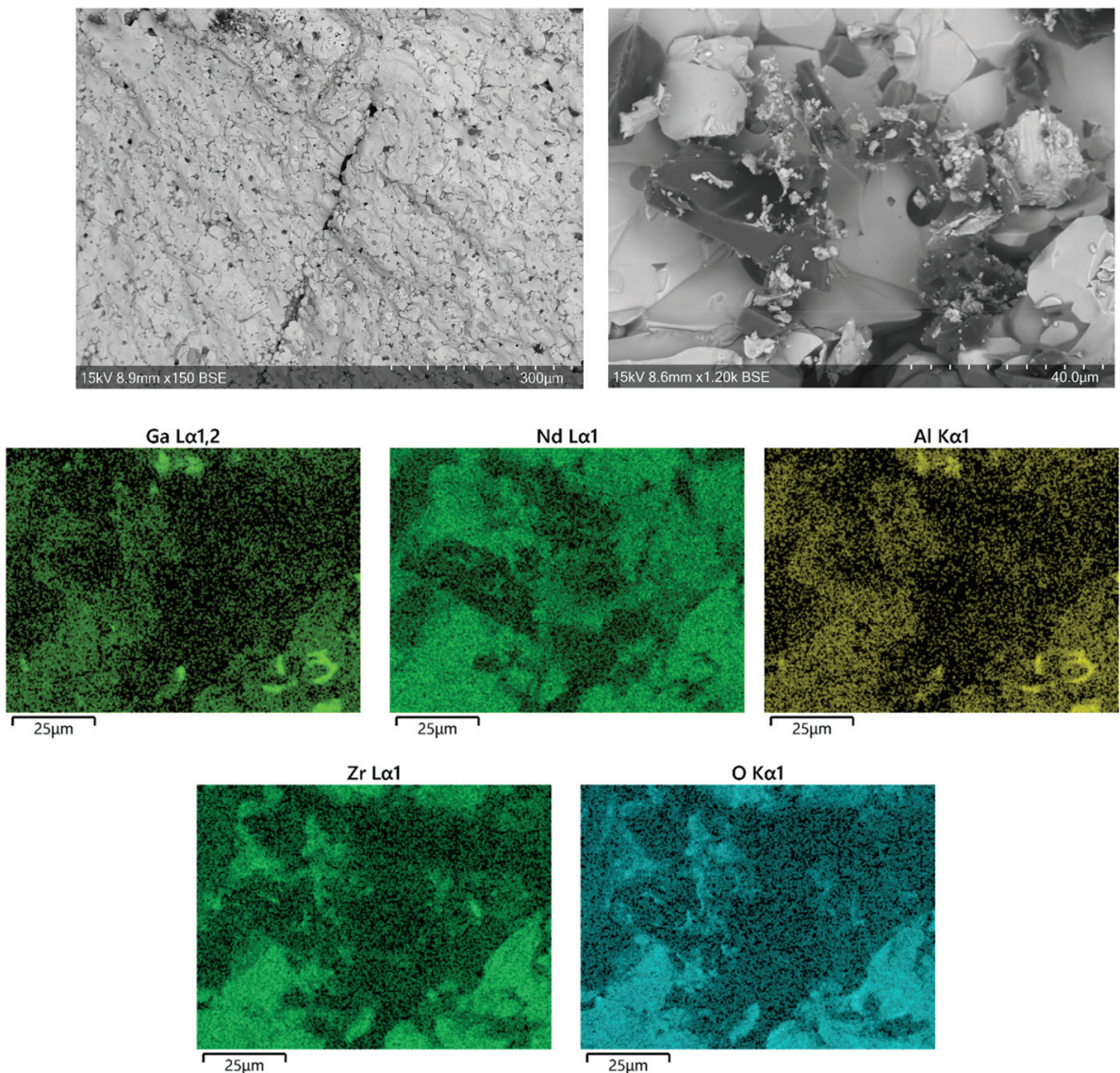

Fig. 16 Side SEM images of Ga-NLZO pellet interior after regeneration. Pellet was measured side on after being broken into fragments. Left image shows potential area where dendrite propagated, and right image (and corresponding EDX) shows some elemental separation as in Fig. 15. Al signal is thought to arise from Al stub, or perhaps $\mathrm{Al} / \mathrm{Li}$ exchange from crucible, as this is the same pellet as in Fig. 3.

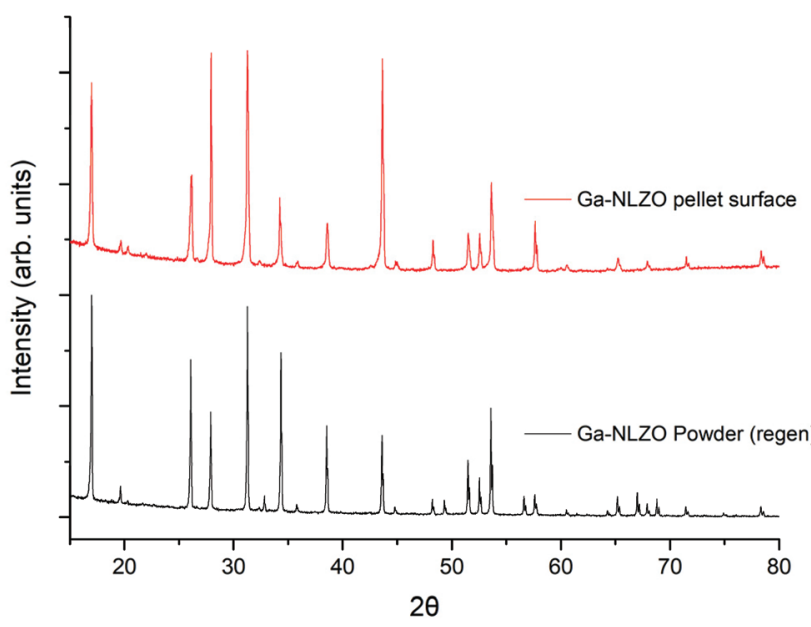

Fig. 17 XRD of Ga-NLZO pellet surface from symmetry cell with Li metal removed (prior to thermal etching), and of the pellet powder taken from re-grinding pellet fragment after regenerating the pellet.

ponding peaks, hence providing some further evidence of a similar reaction occurring at the Ga-NLZO interface. However, some peaks are overlapping with garnet, whereas others are possibly lost in the background, therefore additional work is required to confirm $\mathrm{Ga}-\mathrm{Li}$ eutectic formation more absolutely. But, nonetheless, it is clear that Ga-NLZO garnets present with

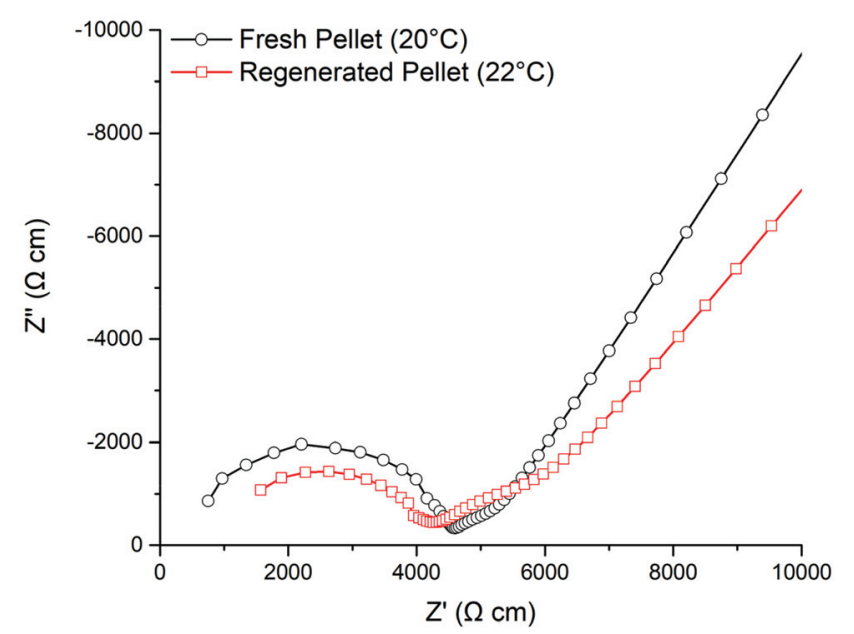

Fig. 18 Impedance spectrum of regeneration pellet (red) overlaid with a fresh pellet (black), illustrating little change outside a small increase in temperature. 
exceptionally low ASR values that are intrinsic to the garnet material, a rare feature.

Finally, the regenerated pellet was sputtered with $\mathrm{Au}$ and the conductivity remeasured to assess the recoverability of the Ga-NLZO garnet materials after a short circuit event. As shown in Fig. 18, the pellet was mostly regenerated back to its original properties, demonstrating a comparable conductivity of $0.2 \mathrm{mS}$ $\mathrm{cm}^{-1}\left(20{ }^{\circ} \mathrm{C}\right)$, with the corresponding XRD of the ground powder displayed in Fig. 17.

\section{Conclusion}

In summary, a range of $\mathrm{Nd}$ based $\mathrm{Li}$ garnet materials have been synthesised for the first time, with Ga-NLZO in particular having room temperature ionic conductivity $\left(\geq 2 \times 10^{-4} \mathrm{~S} \mathrm{~cm}^{-1}\right)$ similar to La based garnets, despite having significantly smaller lattice parameters. Ga-NLZO also appears relatively straightforward to sinter reproducibly, giving pellets with a high density in lower processing times than equivalent La based garnets. Significantly, we have demonstrated that GaNLZO enables very low ASR values in contact with Li metal which remain stable during cell cycling, a feature which is intrinsic to the garnet material itself rather than requiring additional coatings. It is proposed this is due to Ga-Li eutectic formation, which is enhanced by Ga-NLZO due to its much smaller lattice parameters (compared to Ga-LLZO) increasing $\mathrm{Ga}$ exsolution when combined with $\mathrm{Li}$ metal/heating. Somewhat surprisingly despite the very low ASR value, a comparatively low CCD was observed which requires future work to understand fully, but we propose that it relates to the same Ga exsolution process which enables low ASR values. Despite this issue, the high density and low ASR of Ga-NLZO allows it to be utilized in conjunction with a La based garnet as an interfacial modification to overcome the latter's high Li/electrode interfacial resistance, whereupon Ga-NLZO would act as a CI layer between the Li electrode and the garnet electrolyte.

\section{Raw experimental data}

https://doi.org/10.25500/edata.bham.00000716.

\section{Conflicts of interest}

There are no conflicts of interest to declare.

\section{Acknowledgements}

We would like to thank the University of Birmingham for the studentship funding of Mark Stockham and Matthew James, and the EPSRC for funding the GENESIS project (under EP/ R024006/1) and the EPSRC Capital Award for Core Equipment (EP/T02349X/1).

\section{Notes and references}

1 S. Wang, K. Rafiz, J. Liu, Y. Jin and J. Y. S. Lin, Sustainable Energy Fuels, 2020, 4, 2342-2351.

2 B. S. Vishnugopi, F. Hao, A. Verma and P. P. Mukherjee, ACS Appl. Mater. Interfaces, 2020, 12, 23931-23938.

3 M. A. Howard, O. Clemens, K. S. Knight, P. A. Anderson, S. Hafiz, P. M. Panchmatia and P. R. Slater, J. Mater. Chem. A, 2013, 1, 14013-14022.

4 J. M. Tarascon and M. Armand, Nature, 2001, 414, 359.

5 C.-X. Zu and H. Li, Energy Environ. Sci., 2011, 4, 26142624.

6 V. A. Agubra and J. W. Fergus, J. Power Sources, 2014, 268, 153-162.

7 N. Nitta, F. Wu, J. T. Lee and G. Yushin, Mater. Today, 2015, 18, 252-264.

8 M. Winter and R. J. Brodd, Chem. Rev., 2004, 104, 4245-4270.

9 T. Kim, W. Song, D.-Y. Son, L. K. Ono and Y. Qi, J. Mater. Chem. A, 2019, 7, 2942-2964.

10 M. Armand and J. M. Tarascon, Nature, 2008, 451, 652.

11 H. Yang, C. Guo, A. Naveed, J. Lei, J. Yang, Y. Nuli and J. Wang, Energy Storage Mater., 2018, 14, 199-221.

12 L. Wang, Z. Zhou, X. Yan, F. Hou, L. Wen, W. Luo, J. Liang and S. X. Dou, Energy Storage Mater., 2018, 14, 22-48.

13 H. Wu, D. Zhuo, D. Kong and Y. Cui, Nat. Commun., 2014, $5,5193$.

14 Y. Zhu, X. He and Y. Mo, ACS Appl. Mater. Interfaces, 2015, 7, 23685-23693.

15 J. Li, C. Ma, M. Chi, C. Liang and N. J. Dudney, Adv. Energy Mater., 2015, 5, 1401408.

16 H. Liu, X.-B. Cheng, J.-Q. Huang, H. Yuan, Y. Lu, C. Yan, G.-L. Zhu, R. Xu, C.-Z. Zhao, L.-P. Hou, C. He, S. Kaskel and Q. Zhang, ACS Energy Lett., 2020, 5, 833-843.

17 B. Dong, J. Yan, B. Walkley, K. K. Inglis, F. Blanc, S. Hull and A. R. West, Solid State Ionics, 2018, 327, 64-70.

18 V. Thangadurai, S. Narayanan and D. Pinzaru, Chem. Soc. Rev., 2014, 43, 4714-4727.

19 W. D. Richards, L. J. Miara, Y. Wang, J. C. Kim and G. Ceder, Chem. Mater., 2016, 28, 266-273.

20 Y. G. Kim and H. N. G. Wadley, J. Power Sources, 2011, 196, 1371-1377.

21 Y. Zhao and L. L. Daemen, Superionic Conductivity in Lithium-Rich Anti-Perovskites, 2012.

22 Y. Zheng, Y. Yao, J. Ou, M. Li, D. Luo, H. Dou, Z. Li, K. Amine, A. Yu and Z. Chen, Chem. Soc. Rev., 2020, 49, 8790-8839.

23 Y. Zhang, F. Chen, R. Tu, Q. Shen and L. Zhang, J. Power Sources, 2014, 268, 960-964.

24 M. Botros, R. Djenadic, O. Clemens, M. Möller and H. Hahn, J. Power Sources, 2016, 309, 108-115.

25 E. Kazyak, K.-H. Chen, K. N. Wood, A. L. Davis, T. Thompson, A. R. Bielinski, A. J. Sanchez, X. Wang, C. Wang, J. Sakamoto and N. P. Dasgupta, Chem. Mater., 2017, 29, 3785-3792.

26 A. Sharafi, H. M. Meyer, J. Nanda, J. Wolfenstine and J. Sakamoto, J. Power Sources, 2016, 302, 135-139. 
27 L. Cheng, W. Chen, M. Kunz, K. Persson, N. Tamura, G. Chen and M. Doeff, ACS Appl. Mater. Interfaces, 2015, 7, 2073-2081.

28 L. Cheng, C. H. Wu, A. Jarry, W. Chen, Y. Ye, J. Zhu, R. Kostecki, K. Persson, J. Guo, M. Salmeron, G. Chen and M. Doeff, ACS Appl. Mater. Interfaces, 2015, 7, 1764917655.

29 C. Bernuy-Lopez, W. Manalastas, J. M. Lopez del Amo, A. Aguadero, F. Aguesse and J. A. Kilner, Chem. Mater., 2014, 26, 3610-3617.

30 Q. Liu, Z. Geng, C. Han, Y. Fu, S. Li, Y.-b. He, F. Kang and B. Li, J. Power Sources, 2018, 389, 120-134.

31 N. J. Taylor, S. Stangeland-Molo, C. G. Haslam, A. Sharafi, T. Thompson, M. Wang, R. Garcia-Mendez and J. Sakamoto, J. Power Sources, 2018, 396, 314-318.

32 M. P. Stockham, B. Dong, M. S. James, Y. Li, Y. Ding and P. R. Slater, Dalton Trans., 2021, 50, 2364-2374.

33 Y. Jin and P. J. McGinn, J. Power Sources, 2013, 239, 326331.

34 R. H. Brugge, J. A. Kilner and A. Aguadero, Solid State Ionics, 2019, 337, 154-160.

35 H. Duan, H. Zheng, Y. Zhou, B. Xu and H. Liu, Solid State Ionics, 2018, 318, 45-53.

36 A. F. Wells, Structural inorganic chemistry, Clarendon Press, 1984.

37 V. Thangadurai, S. Adams and W. Weppner, Chem. Mater., 2004, 16, 2998-3006.

38 E. J. Cussen and T. W. S. Yip, J. Solid State Chem., 2007, 180, 1832-1839.

39 E. J. Cussen, Chem. Commun., 2006, 412-413, DOI: 10.1039/B514640B.

40 M. P. O'Callaghan, D. R. Lynham, E. J. Cussen and G. Z. Chen, Chem. Mater., 2006, 18, 4681-4689.

41 V. Thangadurai, H. Kaack and W. J. F. Weppner, J. Am. Ceram. Soc., 2004, 86, 437-440.

42 R. Murugan, V. Thangadurai and W. Weppner, Angew. Chem., Int. Ed., 2007, 46, 7778-7781.

43 C. A. Geiger, E. Alekseev, B. Lazic, M. Fisch, T. Armbruster, R. Langner, M. Fechtelkord, N. Kim, T. Pettke and W. Weppner, Inorg. Chem., 2011, 50, 1089-1097.

44 J. Awaka, N. Kijima, K. Kataoka, H. Hayakawa, K.-i. Ohshima and J. Akimoto, J. Solid State Chem., 2010, 183, 180-185.

45 J. Awaka, N. Kijima, H. Hayakawa and J. Akimoto, J. Solid State Chem., 2009, 182, 2046-2052.

46 J. Percival, E. Kendrick, R. I. Smith and P. R. Slater, Dalton Trans., 2009, 5177-5181, DOI: 10.1039/b907331k.

47 B. Dong, S. R. Yeandel, P. Goddard and P. R. Slater, Chem. Mater., 2020, 32, 215-223.

48 R. Wagner, G. J. Redhammer, D. Rettenwander, A. Senyshyn, W. Schmidt, M. Wilkening and G. Amthauer, Chem. Mater., 2016, 28, 1861-1871.

49 J. Percival and P. R. Slater, Solid State Commun., 2007, 142, 355-357.

50 R. Murugan, V. Thangadurai and W. Weppner, Appl. Phys. A, 2008, 91, 615-620.
51 M. A. Howard, O. Clemens, E. Kendrick, K. S. Knight, D. C. Apperley, P. A. Anderson and P. R. Slater, Dalton Trans., 2012, 41, 12048-12053.

52 J. L. Allen, J. Wolfenstine, E. Rangasamy and J. Sakamoto, J. Power Sources, 2012, 206, 315-319.

53 B. Dong, L. L. Driscoll, M. P. Stockham, E. Kendrick and P. R. Slater, Solid State Ionics, 2020, 350, 115317.

54 B. Dong, M. P. Stockham, P. A. Chater and P. R. Slater, Dalton Trans., 2020, 49, 11727-11735.

55 F. M. Pesci, R. H. Brugge, A. K. O. Hekselman, A. Cavallaro, R. J. Chater and A. Aguadero, J. Mater. Chem. A, 2018, 6, 19817-19827.

56 M. A. Howard, O. Clemens, E. Kendrick, K. S. Knight, D. C. Apperley, P. A. Anderson and P. R. Slater, Dalton Trans., 2012, 41, 12048-12053.

57 S. Narayanan and V. Thangadurai, J. Power Sources, 2011, 196, 8085-8090.

58 S. Narayanan, F. Ramezanipour and V. Thangadurai, J. Phys. Chem. C, 2012, 116, 20154-20162.

59 J.-F. Wu, E.-Y. Chen, Y. Yu, L. Liu, Y. Wu, W. K. Pang, V. K. Peterson and X. Guo, ACS Appl. Mater. Interfaces, 2017, 9, 1542-1552.

60 X. Wang, J. Liu, R. Yin, Y. Xu, Y. Cui, L. Zhao and X. Yu, Mater. Lett., 2018, 231, 43-46.

61 J. Gai, E. Zhao, F. Ma, D. Sun, X. Ma, Y. Jin, Q. Wu and Y. Cui, J. Eur. Ceram. Soc., 2018, 38, 1673-1678.

62 N. Janani, C. Deviannapoorani, L. Dhivya and R. Murugan, RSC Adv., 2014, 4, 51228-51238.

63 V. Thangadurai and W. Weppner, J. Am. Ceram. Soc., 2005, 88, 411-418.

64 L. Robben, E. Merzlyakova, P. Heitjans and T. M. Gesing, Acta Crystallogr., Sect. E, 2016, 72, 287-289.

65 Y. Ren, Y. Shen, Y. Lin and C.-W. Nan, Electrochem. Commun., 2015, 57, 27-30.

66 D. Wang, K. Peng, Y. Fu, C. Zhu and Y. Yang, J. Power Sources, 2021, 487, 229421.

67 Y. Zhu, J. G. Connell, S. Tepavcevic, P. Zapol, R. GarciaMendez, N. J. Taylor, J. Sakamoto, B. J. Ingram, L. A. Curtiss, J. W. Freeland, D. D. Fong and N. M. Markovic, Adv. Energy Mater., 2019, 9, 1803440.

68 R. H. Brugge, F. M. Pesci, A. Cavallaro, C. Sole, M. A. Isaacs, G. Kerherve, R. S. Weatherup and A. Aguadero, J. Mater. Chem. A, 2020, 8, 14265-14276.

69 J. Saint, M. Morcrette, D. Larcher and J. M. Tarascon, Solid State Ionics, 2005, 176, 189-197.

70 J. Sangster and A. D. Pelton, J. Phase Equilib., 1991, 12, 33-36.

71 H. Okamoto, J. Phase Equilib. Diffus., 2006, 27, 200-200.

72 H. Xie, K.-S. Park, J. Song and J. B. Goodenough, Electrochem. Commun., 2012, 19, 135-137.

73 M. A. Howard, O. Clemens, A. S. Parvathy, P. A. Anderson and P. R. Slater, J. Alloys Compd., 2016, 670, 78-84.

74 B. Toby and R. Dreele, J. Appl. Crystallogr., 2013, 46, 544549.

75 R. H. Brugge, A. K. O. Hekselman, A. Cavallaro, F. M. Pesci, R. J. Chater, J. A. Kilner and A. Aguadero, Chem. Mater., 2018, 30, 3704-3713. 
76 G. Larraz, A. Orera and M. L. Sanjuán, J. Mater. Chem. A, 2013, 1, 11419-11428.

77 C. Galven, J. Dittmer, E. Suard, F. Le Berre and M.-P. Crosnier-Lopez, Chem. Mater., 2012, 24, 3335-3345.

78 J. Percival, D. Apperley and P. R. Slater, Solid State Ionics, 2008, 179, 1693-1696.

79 H. Peng, Y. Zhang, L. Li and L. Feng, Solid State Ionics, 2017, 304, 71-74.

80 I. P. Roof, M. D. Smith, E. J. Cussen and H.-C. zur Loye, J. Solid State Chem., 2009, 182, 295-300.

81 F. M. Pesci, A. Bertei, R. H. Brugge, S. P. Emge, A. K. O. Hekselman, L. E. Marbella, C. P. Grey and A. Aguadero, ACS Appl. Mater. Interfaces, 2020, 12, 3280632816.

82 A. Gupta, R. Murugan, M. P. Paranthaman, Z. Bi, C. A. Bridges, M. Nakanishi, A. P. Sokolov, K. S. Han, E. W. Hagaman, H. Xie, C. B. Mullins and J. B. Goodenough, J. Power Sources, 2012, 209, 184-188.

83 Y. Wang, P. Yan, J. Xiao, X. Lu, J.-G. Zhang and V. L. Sprenkle, Solid State Ionics, 2016, 294, 108-115.

84 M. P. Stockham, B. Dong, Y. Ding, Y. Li and P. R. Slater, Dalton Trans., 2020, 49, 10349-10359.

85 S. Song, B. Chen, Y. Ruan, J. Sun, L. Yu, Y. Wang and J. Thokchom, Electrochim. Acta, 2018, 270, 501-508.

86 M. P. Stockham, B. Dong, Y. Ding, Y. Li and P. R. Slater, Dalton Trans., 2020, 49, 10349-10359.

87 G. V. Alexander, S. Patra, S. V. Sobhan Raj, M. K. Sugumar, M. M. Ud Din and R. Murugan, J. Power Sources, 2018, 396, 764-773.

88 C.-L. Tsai, V. Roddatis, C. V. Chandran, Q. Ma, S. Uhlenbruck, M. Bram, P. Heitjans and O. Guillon, ACS Appl. Mater. Interfaces, 2016, 8, 10617-10626.

89 N. C. Rosero-Navarro, R. Kajiura, R. Jalem, Y. Tateyama, A. Miura and K. Tadanaga, ACS Appl. Energy Mater., 2020, 3, 5533-5541.

90 B. Liu, Y. Gong, K. Fu, X. Han, Y. Yao, G. Pastel, C. Yang, H. Xie, E. D. Wachsman and L. Hu, ACS Appl. Mater. Interfaces, 2017, 9, 18809-18815.

91 W. Zhou, Y. Zhu, N. Grundish, X. Sen, S. Wang, Y. You, N. Wu, J. Gao, Z. Cui, Y. Li and J. B. Goodenough, Nano Energy, 2018, 53, 926-931.
92 B. Xu, W. Li, H. Duan, H. Wang, Y. Guo, H. Li and H. Liu, J. Power Sources, 2017, 354, 68-73.

93 A. Sharafi, E. Kazyak, A. L. Davis, S. Yu, T. Thompson, D. J. Siegel, N. P. Dasgupta and J. Sakamoto, Chem. Mater., 2017, 29, 7961-7968.

94 Y. Shao, H. Wang, Z. Gong, D. Wang, B. Zheng, J. Zhu, Y. Lu, Y.-S. Hu, X. Guo, H. Li, X. Huang, Y. Yang, C.-W. Nan and L. Chen, ACS Energy Lett., 2018, 3, 12121218.

95 W. Luo, Y. Gong, Y. Zhu, K. K. Fu, J. Dai, S. D. Lacey, C. Wang, B. Liu, X. Han, Y. Mo, E. D. Wachsman and L. Hu, J. Am. Chem. Soc., 2016, 138, 12258-12262.

96 W. Luo, Y. Gong, Y. Zhu, Y. Li, Y. Yao, Y. Zhang, K. Fu, G. Pastel, C.-F. Lin, Y. Mo, E. D. Wachsman and L. Hu, Adv. Mater., 2017, 29, 1606042.

97 K. Fu, Y. Gong, B. Liu, Y. Zhu, S. Xu, Y. Yao, W. Luo, C. Wang, S. D. Lacey, J. Dai, Y. Chen, Y. Mo, E. Wachsman and L. Hu, Sci. Adv., 2017, 3, e1601659.

98 R. Sudo, Y. Nakata, K. Ishiguro, M. Matsui, A. Hirano, Y. Takeda, O. Yamamoto and N. Imanishi, Solid State Ionics, 2014, 262, 151-154.

99 C. Wang, Y. Gong, B. Liu, K. Fu, Y. Yao, E. Hitz, Y. Li, J. Dai, S. Xu, W. Luo, E. D. Wachsman and L. Hu, Nano Lett., 2017, 17, 565-571.

100 W. Feng, X. Dong, P. Li, Y. Wang and Y. Xia, J. Power Sources, 2019, 419, 91-98.

101 M. He, Z. Cui, C. Chen, Y. Li and X. Guo, J. Mater. Chem. A, 2018, 6, 11463-11470.

102 J.-F. Wu, B.-W. Pu, D. Wang, S.-Q. Shi, N. Zhao, X. Guo and X. Guo, ACS Appl. Mater. Interfaces, 2019, 11, 898-905.

103 Y. Li, X. Chen, A. Dolocan, Z. Cui, S. Xin, L. Xue, H. Xu, K. Park and J. B. Goodenough, J. Am. Chem. Soc., 2018, 140, 6448-6455.

104 F. Flatscher, M. Philipp, S. Ganschow, H. M. R. Wilkening and D. Rettenwander, J. Mater. Chem. A, 2020, 8, 1578215788.

105 R. Hongahally Basappa, T. Ito, T. Morimura, R. Bekarevich, K. Mitsuishi and H. Yamada, J. Power Sources, 2017, 363, 145-152.

106 X. Huang, Y. Lu, H. Guo, Z. Song, T. Xiu, M. E. Badding and Z. Wen, ACS Appl. Energy Mater., 2018, 1, 5355-5365. 